\title{
Quantum Calculations of pKa values for Some Amine Compounds
}

\author{
Ammar A. Ibrahim ${ }^{1 *}$ \\ Entesar A. Sulliman ${ }^{2}$ \\ Simaa M. Daood ${ }^{1}$
}

${ }^{1}$ Department of Chemistry, College of Science, University of Mosul, Iraq

${ }^{2}$ Al-Noor University College, ammar74@uomosul.edu.iq

\begin{abstract}
The theoretical calculations methods DFT/STO-3G, DFT/6-31G, DFT/6$31 \mathrm{G}(\mathrm{d}, \mathrm{p}), \mathrm{HF} / \mathrm{STO}-3 \mathrm{G}, \mathrm{HF} / 6-31 \mathrm{G}$ and HF/6-31G(d,p) are used to determination the physic-chemical parameters for some aliphatic amine. This data was used to predict $\mathrm{pKa}$ values. Two methods of statistics are used to evaluate the pKa depends on the theoretical data. The first statistical method is used $\Delta \mathrm{G}$ data to determine the pKa. The second statistical method depends on the enter all the thermodynamic and physic-chemical values in the equation using multiple linear regression. Finally, the two methods are compared with experimental.
\end{abstract}

Keywords: DFT, HF, pKa, Theoretical calculation, HOMO, LUMO

\section{Introduction:}

Amines define as a strong base which were converted to protonated form at using dilute acid solutions or in aqueous media:

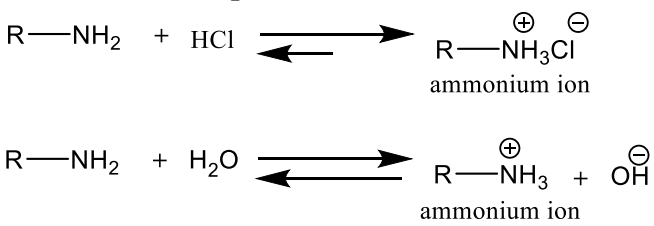

Also, amines compounds are known as the strongest base because of the free non-bonding electron on the nitrogen atom. This property makes the amine group accepted the proton from other compounds and converted to ammonium ion. The cycle to calculate the thermodynamic parameters of the compound can be determined depending on the equation of the dissociation for acid:

$$
\mathrm{HA}^{+}\left({ }_{(\mathrm{aq})} \rightarrow \mathrm{A}_{(\mathrm{aq})}+\mathrm{H}^{+}(\mathrm{aq})\right.
$$

So, the equation is became : $\Delta \mathrm{G}_{\text {gas }}=\mathrm{G}_{\text {gas. }}\left(\mathrm{H}^{+}\right)+\mathrm{G}_{\text {gas. }}(\mathrm{A})-\mathrm{G}_{\text {gas. }}\left(\mathrm{HA}^{+}\right)$for gas state. And, $\Delta \mathrm{G}_{\text {sol }}$. $=\Delta \mathrm{G}_{\text {sol. }}\left(\mathrm{H}^{+}\right)+\Delta \mathrm{G}_{\text {sol. }}(\mathrm{A})-\Delta \mathrm{G}_{\text {sol. }}\left(\mathrm{HA}^{+}\right)$for the solvation state. Value of $\left(\mathrm{G}_{\text {gas. }} \mathrm{H}^{+}\right)$is taken as (-6.28) $\mathrm{kcal} / \mathrm{mol}[1,2]$, while $\left(\Delta \mathrm{G}_{\mathrm{sol}} . \mathrm{H}^{+}\right)$is taken different value at different papers like (-254), (-261.4), (-264.61), (-265.6), and (-267.9) in kcal/mol[3-6]. So, we choose the value (-267.9) $\mathrm{kca} / \mathrm{mol}$ in the statics.

Because of the importance of the nitrogen atoms in life especially in caffeine, amino and nucleic acids, nicotine and hormones. Many compounds are synthesis or studied like drugs[7,8], heterocyclic[9], biological acticity[10], antioxidant[11,12], and complex[13]. Also, azo-compounds which containing series of $(-\mathrm{N}=\mathrm{N}-)$ were calculated theoretically[14] and electrochemically[15]. The physico-chemical parameters are used to estimate the pKa-values by using computational chemistry depending on the theoretical calculations. It's used in the accuracy of drugs [16] and its metabolism[17].

The pKa can be determined depending on the different descriptors like charge, hardness, steric energy, LUMO, HOMO, etc[18]. The dissociation constant ( $\mathrm{pKa}$ ) are accurate for many pharmaceutical antibiotic compounds (amoxicillin, ampicillin, penicillin, sulfonamides)[19]. The shift of pKa in nucleotides on the RNA compounds has been determined using the equation of Poisson Boltzmann[20]. Drugs of substituted carbo-xamides structure contain many nitrogen atoms were studied their basicities theoretically using (DFT) methods[21]. Also, UV spectroscopic instrumental was used to evaluate the pKa parameters[22].

Density function theory(DFT/B3LYP) was used for a series of pyridinium and anilinum ion at a different solvent to predicted the ( $\mathrm{pKa}$ ) parameters[23]. The thermodynamic-cycles for a reaction that 
depends on the gas and salvation phase were calculated to find the (pKa) parameters[24,25]. Finally, the theoretical calculation is an important tool for the prediction of the ionization potential[26].

AM1 and PM3 methods were used to predict the pKa for series of aniline compound depend on the linear correlation between the experimental pKa with hardness rely on the LUMO and HOMO parameters[27]. Substituted of pyridine which is used as anti viral[28], anti cancer[29] and anti inflammatory[30] have been determined by theoretical calculations upon by WB97XD and B3LYP at different basis set in the gas phase. While AM1 and PM5 were used to study the salvation effect to find the $\mathrm{pKa}$ for a series of protonation of pyridine[31].

\section{Experimental:}

Gaussian 03 program was used in the determination and calculations of the compounds. These compounds have been optimized in the gas, ion, gas-solvation and ion-solvation using Hartree-Fock and density functional theory (B3LYP) at different basis set STO-3G, 6-31G and 6-31G(d,p). Later, CPCM method was used in the solvation in water media.

Table 1. The number, names and the experimental $\mathrm{pKa}$ values for compounds

\begin{tabular}{llllll}
\hline No. & Compounds & Exp. pKa & No. & Compounds & Exp. pKa \\
\hline 1 & Benzylamine & 9.34 & 7 & n-propylamine & 10.53 \\
2 & Dimethylamine & 10.60 & 8 & t-butylamine & 10.45 \\
3 & di-n-butylamine & 11.25 & 9 & Triethylamine & 10.76 \\
4 & Ethylamine & 10.63 & 10 & Trimethylamine & 9.76 \\
5 & Methylamine & 10.62 & 11 & tri-n-propylamine & 10.65 \\
6 & n-butylamine & 10.59 & & & \\
\hline
\end{tabular}

\section{Results and Discussion:}

In this research, we try to choose two methods to the determination of pKa values. The first one depends on the calculation of the compounds in the gas phase and in salvation media. The seconds method is evaluated by all the parameters and used to predict by the statistical program. Also, chemical potential, softness and hardness were determined depending on the HOMO and LUMO parameters.

Chemical potential $=(\mathrm{LUMO}+\mathrm{HOMO}) / 2$

Hardness $=($ LUMO-HOMO $) / 2$

Softness $=1$ - hardness

Electro-philicity $=(\text { Chemical potential })^{2} /(2 \times$ hardness $)$

\subsection{The First Method:}

Used the methods of calculations DFT/STO-3G, DFT/6-31G, DFT/6-31G(d,p), HF/STO-3G, $\mathrm{HF} / 6-31 \mathrm{G}, \mathrm{HF} / 6-31 \mathrm{G}(\mathrm{d}, \mathrm{p})$ in the gas and in salvation phase. The free energy factor $(\Delta \mathrm{G})$ was used to found the pKa practically using the methylamine as a reference $(\mathrm{pKa}=10.62)$. Later, the $\Delta \mathrm{G}_{\text {total }}$ values were represented by the summation between the compound in the gas and solvation media which were shown in tables (2-7). 
Table (2): Free energy for neutral and ion in gas and solvation using DFT/STO-3G

\begin{tabular}{lllllllll}
\hline DFT/STO-3G & $\begin{array}{l}\Delta \mathrm{G} \text { Gas } \\
\text { a.u }\end{array}$ & $\begin{array}{l}\text { Ion } \\
\text { a.u }\end{array}$ & $\begin{array}{l}\Delta \mathrm{G} \text { Solv. } \\
\text { a.u }\end{array}$ & $\begin{array}{l}\text { Ion } \\
\text { a.u }\end{array}$ & $\begin{array}{l}\Delta \mathrm{G} \text { (gas+sol.) } \\
\text { kcal.mol }\end{array}$ & $\begin{array}{l}\mathrm{pKa} \\
\text { Calc. }\end{array}$ & $\begin{array}{l}\mathrm{pKa} \\
\text { Exp. }\end{array}$ & $\begin{array}{l}\Delta \mathrm{pKa} \\
\text { Calc.-Exp. }\end{array}$ \\
\hline 1 & 0.12334 & 0.13766 & 0.12389 & 0.13586 & 1.1791 & 9.44 & 9.34 & 0.10 \\
2 & 0.07486 & 0.08891 & 0.07465 & 0.08797 & 0.5033 & 10.12 & 10.60 & -0.48 \\
3 & 0.24930 & 0.26318 & 0.24840 & 0.26366 & -0.6124 & 11.23 & 11.25 & -0.02 \\
4 & 0.07490 & 0.08932 & 0.07461 & 0.08837 & -0.0088 & 10.63 & 10.63 & 0.00 \\
5 & 0.04606 & 0.06058 & 0.04582 & 0.05947 & 0.0000 & 10.62 & 10.62 & 0.00 \\
6 & 0.13312 & 0.14748 & 0.13269 & 0.14645 & 0.0264 & 10.59 & 10.59 & 0.00 \\
7 & 0.10401 & 0.11834 & 0.10362 & 0.11736 & 0.0609 & 10.56 & 10.53 & 0.03 \\
8 & 0.13265 & 0.14671 & 0.13249 & 0.14622 & 0.2391 & 10.38 & 10.45 & -0.07 \\
9 & 0.19072 & 0.20428 & 0.19064 & 0.20566 & -0.2579 & 10.88 & 10.76 & 0.12 \\
10 & 0.10378 & 0.11735 & 0.10373 & 0.11669 & 1.0329 & 9.59 & 9.76 & -0.17 \\
11 & 0.27767 & 0.29170 & 0.27740 & 0.29099 & 0.3464 & 10.27 & 10.65 & -0.38 \\
\hline
\end{tabular}

Table (3): Free energy for neutral and ion in gas and solvation using DFT/6-31G

\begin{tabular}{lllllllll}
\hline DFT/6-31G & $\begin{array}{l}\Delta \mathrm{G} \mathrm{Gas} \\
\text { a.u }\end{array}$ & $\begin{array}{l}\text { Ion } \\
\text { a.u }\end{array}$ & $\begin{array}{l}\Delta \mathrm{G} \text { Solv. } \\
\text { a.u }\end{array}$ & $\begin{array}{l}\text { Ion } \\
\text { a.u }\end{array}$ & $\begin{array}{l}\Delta \mathrm{G} \text { (gas+sol.) } \\
\text { kcal.mol }\end{array}$ & $\begin{array}{l}\mathrm{pKa} \\
\text { Calc. }\end{array}$ & $\begin{array}{l}\mathrm{pKa} \\
\text { Exp. }\end{array}$ & $\begin{array}{l}\Delta \mathrm{pKa} \\
\text { Calc.-Exp. }\end{array}$ \\
\hline 1 & 0.11564 & 0.13090 & 0.11360 & 0.13002 & -0.4374 & 11.06 & 9.34 & 1.72 \\
2 & 0.06740 & 0.08330 & 0.06705 & 0.08201 & 0.0734 & 10.55 & 10.60 & -0.05 \\
3 & 0.22639 & 0.24221 & 0.22474 & 0.24164 & -1.0906 & 11.71 & 11.25 & 0.46 \\
4 & 0.06760 & 0.08333 & 0.06712 & 0.08200 & 0.2353 & 10.38 & 10.63 & -0.25 \\
5 & 0.04113 & 0.05713 & 0.04075 & 0.05573 & 0.0000 & 10.62 & 10.62 & 0.00 \\
6 & 0.12064 & 0.13635 & 0.11992 & 0.13472 & 0.2887 & 10.33 & 10.59 & -0.26 \\
7 & 0.09406 & 0.10983 & 0.09344 & 0.10831 & 0.2058 & 10.41 & 10.53 & -0.12 \\
8 & 0.12035 & 0.13561 & 0.11988 & 0.13465 & 0.5917 & 10.03 & 10.45 & -0.42 \\
9 & 0.17349 & 0.18900 & 0.17334 & 0.18880 & 0.0038 & 10.62 & 10.76 & -0.14 \\
10 & 0.09376 & 0.10952 & 0.09372 & 0.10856 & 0.2328 & 10.39 & 9.76 & 0.63 \\
11 & 0.25301 & 0.26899 & 0.25276 & 0.26667 & 0.6815 & 9.94 & 10.65 & -0.71 \\
\hline
\end{tabular}

Table (4): Free energy for neutral and ion in gas and solvation using DFT/6-31G(d,p)

\begin{tabular}{lllllllll}
\hline DFT/6-31G(d,p) & $\begin{array}{l}\Delta \mathrm{G} \text { Gas } \\
\text { a.u }\end{array}$ & $\begin{array}{l}\text { Ion } \\
\text { a.u }\end{array}$ & $\begin{array}{l}\Delta \mathrm{G} \text { Solv. } \\
\text { a.u }\end{array}$ & $\begin{array}{l}\text { Ion } \\
\text { a.u }\end{array}$ & $\begin{array}{l}\Delta \mathrm{G} \text { (gas+sol.) } \\
\text { kcal.mol }\end{array}$ & $\begin{array}{l}\mathrm{pKa} \\
\text { Calc. }\end{array}$ & $\begin{array}{l}\mathrm{pKa} \\
\text { Exp. }\end{array}$ & $\begin{array}{l}\Delta \mathrm{pKa} \\
\text { Calc.-Exp. }\end{array}$ \\
\hline 1 & 0.11457 & 0.12908 & 0.11438 & 0.12589 & 1.9296 & 8.69 & 9.34 & -0.65 \\
2 & 0.06725 & 0.08248 & 0.06681 & 0.08115 & -0.2918 & 10.91 & 10.60 & 0.31 \\
3 & 0.22490 & 0.23995 & 0.22329 & 0.23934 & -1.2418 & 11.86 & 11.25 & 0.61 \\
4 & 0.06750 & 0.08227 & 0.06684 & 0.08090 & 0.1782 & 10.44 & 10.63 & -0.19 \\
5 & 0.04130 & 0.05629 & 0.04075 & 0.05486 & 0.0000 & 10.62 & 10.62 & 0.00 \\
6 & 0.12007 & 0.13485 & 0.11912 & 0.13319 & 0.1663 & 10.45 & 10.59 & -0.14 \\
7 & 0.09373 & 0.10853 & 0.09291 & 0.10699 & 0.1431 & 10.48 & 10.53 & -0.05 \\
8 & 0.11965 & 0.13407 & 0.11912 & 0.13306 & 0.4750 & 10.14 & 10.45 & -0.31 \\
9 & 0.17194 & 0.18694 & 0.17152 & 0.18675 & -0.7028 & 11.32 & 10.76 & 0.56 \\
10 & 0.09331 & 0.10869 & 0.09320 & 0.10776 & -0.5215 & 11.14 & 9.76 & 1.38 \\
11 & 0.25123 & 0.26649 & 0.25056 & 0.26654 & -1.3422 & 11.96 & 10.65 & 1.31 \\
\hline
\end{tabular}

Table (5): Free energy for neutral and ion in gas and solvation using HF/STO-3G

\begin{tabular}{lllllllll}
\hline HF/STO-3G & $\begin{array}{l}\Delta \mathrm{G} \text { Gas } \\
\text { a.u }\end{array}$ & $\begin{array}{l}\text { Ion } \\
\text { a.u }\end{array}$ & $\begin{array}{l}\Delta \mathrm{G} \text { Solv. } \\
\text { a.u }\end{array}$ & $\begin{array}{l}\text { Ion } \\
\text { a.u }\end{array}$ & $\begin{array}{l}\Delta \mathrm{G} \text { (gas+sol.) } \\
\text { kcal.mol }\end{array}$ & $\begin{array}{l}\mathrm{pKa} \\
\text { Calc. }\end{array}$ & $\begin{array}{l}\mathrm{pKa} \\
\text { Exp. }\end{array}$ & $\begin{array}{l}\Delta \mathrm{pKa} \\
\text { Calc.-Exp. }\end{array}$ \\
\hline 1 & 0.13829 & 0.15347 & 0.13919 & 0.15158 & 1.1552 & 9.46 & 9.34 & 0.12 \\
2 & 0.08439 & 0.09910 & 0.08423 & 0.09837 & 0.3476 & 10.27 & 10.60 & -0.33 \\
3 & 0.27617 & 0.29084 & 0.27551 & 0.29151 & -0.7875 & 11.41 & 11.25 & 0.16 \\
4 & 0.08458 & 0.09958 & 0.08436 & 0.09875 & 0.0082 & 10.61 & 10.63 & -0.02 \\
5 & 0.05275 & 0.06783 & 0.05256 & 0.06689 & 0.0000 & 10.62 & 10.62 & 0.00 \\
6 & 0.14862 & 0.16360 & 0.14828 & 0.16268 & 0.0226 & 10.60 & 10.59 & 0.01 \\
7 & 0.11660 & 0.13159 & 0.11631 & 0.13073 & -0.0025 & 10.62 & 10.53 & 0.09 \\
8 & 0.14820 & 0.16297 & 0.14807 & 0.16253 & 0.1117 & 10.51 & 10.45 & 0.06 \\
9 & 0.21177 & 0.22631 & 0.21144 & 0.22794 & -1.0247 & 11.64 & 10.76 & 0.88 \\
10 & 0.11614 & 0.13050 & 0.11610 & 0.13007 & 0.6790 & 9.94 & 9.76 & 0.18 \\
11 & 0.30811 & 0.32288 & 0.30779 & 0.32219 & 0.1519 & 10.47 & 10.65 & -0.18 \\
\hline
\end{tabular}


Table (6): Free energy for neutral and ion in gas and solvation using HF/6-31G

\begin{tabular}{lllllllll}
\hline HF/6-31G & $\begin{array}{l}\Delta \mathrm{G} \text { Gas } \\
\text { a.u }\end{array}$ & $\begin{array}{l}\text { Ion } \\
\text { a.u }\end{array}$ & $\begin{array}{l}\Delta \mathrm{G} \text { Solv. } \\
\text { a.u }\end{array}$ & $\begin{array}{l}\text { Ion } \\
\text { a.u }\end{array}$ & $\begin{array}{l}\Delta \mathrm{G} \text { (gas+sol.) } \\
\text { kcal. mol }\end{array}$ & $\begin{array}{l}\text { pKa } \\
\text { Calc. }\end{array}$ & $\begin{array}{l}\text { pKa } \\
\text { Exp. }\end{array}$ & $\begin{array}{l}\Delta \mathrm{pKa} \\
\text { Calc.-Exp. }\end{array}$ \\
\hline 1 & 0.12657 & 0.14285 & 0.12634 & 0.13863 & 2.5822 & 8.04 & 9.34 & -1.30 \\
2 & 0.07353 & 0.09028 & 0.07325 & 0.08907 & 0.0646 & 10.56 & 10.60 & -0.04 \\
3 & 0.24374 & 0.26033 & 0.24238 & 0.25971 & -0.7794 & 11.40 & 11.25 & 0.15 \\
4 & 0.07383 & 0.09043 & 0.07339 & 0.08911 & 0.2215 & 10.40 & 10.63 & -0.23 \\
5 & 0.04544 & 0.06228 & 0.04512 & 0.06095 & 0.0000 & 10.62 & 10.62 & 0.00 \\
6 & 0.13054 & 0.14721 & 0.12988 & 0.14552 & 0.2272 & 10.39 & 10.59 & -0.20 \\
7 & 0.10216 & 0.11883 & 0.10160 & 0.11731 & 0.1795 & 10.44 & 10.53 & -0.09 \\
8 & 0.13041 & 0.14670 & 0.13004 & 0.14571 & 0.4462 & 10.17 & 10.45 & -0.28 \\
9 & 0.18775 & 0.20402 & 0.18734 & 0.20027 & 2.1775 & 8.44 & 10.76 & -2.32 \\
10 & 0.10174 & 0.11838 & 0.10166 & 0.11753 & 0.1060 & 10.51 & 9.76 & 0.75 \\
11 & 0.27289 & 0.28959 & 0.27235 & 0.28786 & 0.2880 & 10.33 & 10.65 & -0.32 \\
\hline
\end{tabular}

Table (7): Free energy for neutral and ion in gas and solvation using HF/6-31G(d,p)

\begin{tabular}{lllllllll}
\hline HF/6-31G(d,p) & $\begin{array}{l}\Delta \mathrm{G} \text { Gas } \\
\text { a.u }\end{array}$ & $\begin{array}{l}\text { Ion } \\
\text { a.u }\end{array}$ & $\begin{array}{l}\Delta \mathrm{G} \text { Solv. } \\
\text { a.u }\end{array}$ & $\begin{array}{l}\text { Ion } \\
\text { a.u }\end{array}$ & $\begin{array}{l}\Delta \mathrm{G} \text { (gas+sol.) } \\
\text { kcal.mol }\end{array}$ & $\begin{array}{l}\mathrm{pKa} \\
\text { Calc. }\end{array}$ & $\begin{array}{l}\mathrm{pKa} \\
\text { Exp. }\end{array}$ & $\begin{array}{l}\Delta \mathrm{pKa} \\
\text { Calc.-Exp. }\end{array}$ \\
\hline 1 & 0.12503 & 0.14065 & 0.12478 & 0.13957 & 0.3263 & 10.29 & 9.34 & 0.95 \\
2 & 0.07344 & 0.08957 & 0.07302 & 0.08834 & -0.3282 & 10.95 & 10.60 & 0.35 \\
3 & 0.24206 & 0.25817 & 0.24030 & 0.25751 & -1.5060 & 12.13 & 11.25 & 0.88 \\
4 & 0.07371 & 0.08940 & 0.07305 & 0.08806 & 0.1387 & 10.48 & 10.63 & -0.15 \\
5 & 0.04564 & 0.06150 & 0.04509 & 0.06016 & 0.0000 & 10.62 & 10.62 & 0.00 \\
6 & 0.12996 & 0.14574 & 0.12902 & 0.14402 & 0.0879 & 10.53 & 10.59 & -0.06 \\
7 & 0.10181 & 0.11759 & 0.10099 & 0.11605 & 0.0621 & 10.56 & 10.53 & 0.03 \\
8 & 0.12979 & 0.14527 & 0.12925 & 0.14423 & 0.2962 & 10.32 & 10.45 & -0.13 \\
9 & 0.18625 & 0.20216 & 0.18571 & 0.20288 & -1.3523 & 11.97 & 10.76 & 1.21 \\
10 & 0.10244 & 0.11772 & 0.10128 & 0.11691 & 0.0176 & 10.60 & 9.76 & 0.84 \\
11 & 0.27077 & 0.28719 & 0.27004 & 0.28500 & -0.2780 & 10.90 & 10.65 & 0.25 \\
\hline
\end{tabular}

Table (8): Experimental and predicted $\mathrm{pKa}$ at different methods and basis set

\begin{tabular}{|c|c|c|c|c|c|c|c|}
\hline Compounds & $\begin{array}{l}\mathrm{pKa} \\
\text { Exp. }\end{array}$ & $\begin{array}{l}\text { DFT/STO-3G } \\
\text { Calc. }\end{array}$ & $\begin{array}{l}\text { DFT/6-31G } \\
\text { Calc. }\end{array}$ & $\begin{array}{l}\text { DFT/6-31G(d,p) } \\
\text { Calc. }\end{array}$ & $\begin{array}{l}\text { HF/STO-3G } \\
\text { Calc. }\end{array}$ & $\begin{array}{l}\mathrm{HF} / 6-31 \mathrm{G} \\
\text { Calc. }\end{array}$ & $\begin{array}{l}\mathrm{HF} / 6-31 \mathrm{G}(\mathrm{d}, \mathrm{p}) \\
\text { Calc. }\end{array}$ \\
\hline 1 & 9.34 & 9.44 & $11.06^{*}$ & 8.69 & 9.46 & 8.04 & $10.29 *$ \\
\hline 2 & 10.6 & $10.12 *$ & 10.55 & 10.91 & 10.27 & 10.56 & 10.95 \\
\hline 3 & 11.25 & 11.23 & 11.71 & 11.86 & 11.41 & 11.40 & 12.13 \\
\hline 4 & 10.63 & 10.63 & 10.38 & 10.44 & 10.61 & 10.40 & 10.48 \\
\hline 5 & 10.62 & 10.62 & 10.62 & 10.62 & 10.62 & 10.62 & 10.62 \\
\hline 6 & 10.59 & 10.59 & 10.33 & 10.45 & 10.60 & 10.39 & 10.53 \\
\hline 7 & 10.53 & 10.56 & 10.41 & 10.48 & 10.62 & 10.44 & 10.56 \\
\hline 8 & 10.45 & 10.38 & 10.03 & 10.14 & 10.51 & 10.17 & 10.32 \\
\hline 9 & 10.76 & 10.88 & 10.62 & 11.32 & $11.64 *$ & $8.44 *$ & $11.97 *$ \\
\hline 10 & 9.76 & 9.59 & $10.39 *$ & $11.14 *$ & 9.94 & $10.51 *$ & $10.60 *$ \\
\hline 11 & 10.65 & $10.27^{*}$ & $9.94 *$ & $11.96 *$ & 10.47 & 10.33 & 10.90 \\
\hline $\mathrm{R}\left(\mathrm{R}^{*}\right)$ & & $0.932(0.989)$ & $0.125(0.972)$ & $0.702(0.963)$ & $0.863(0.954)$ & $0.569(0.988)$ & $0.631(0.959)$ \\
\hline St. error $(*)$ & & $0.194(0.089)$ & $0.532(0.063)$ & $0.382(0.144)$ & $0.270(0.167)$ & $0.441(0.081)$ & $0.415(0.074)$ \\
\hline Fisher Value $(*)$ & & $59.5(306.55)$ & $0.14(101.63)$ & 8.74 (89.98) & $26.34(80.80)$ & $4.313(290.29)$ & $5.96(69.06)$ \\
\hline
\end{tabular}

\subsection{The Second Method:}

This method is used the parameters which were calculated theoretically. All the data entered in the statistical equations. The experimental values of $\mathrm{pKa}$ were made as a dependent factor, while all data were independent factors. 
Table (9): Physico-chemical parameters in the gas phase using HF/STO-3G

\begin{tabular}{llllllllll}
\hline $\begin{array}{l}\text { HF/STO-3G } \\
\text { Gas Phase }\end{array}$ & HOMO & LUMO & $\begin{array}{l}\text { Zero } \\
\text { energy }\end{array}$ & $\begin{array}{l}\text { Thermal } \\
\text { energy }\end{array}$ & $\begin{array}{l}\text { Thermal } \\
\text { enthalpy }\end{array}$ & $\begin{array}{l}\text { Total } \\
\text { energy }\end{array}$ & C.V & S & HF \\
\hline 1 & -0.27081 & 0.27041 & 0.16995 & 0.17648 & 0.17743 & 110.74 & 23.61 & 82.36 & -320.78 \\
2 & -0.30687 & 0.59349 & 0.10977 & 0.11395 & 0.11489 & 71.50 & 12.43 & 64.20 & -132.61 \\
3 & -0.30062 & 0.54071 & 0.31474 & 0.32625 & 0.32720 & 204.73 & 37.96 & 107.38 & -364.09 \\
4 & -0.32525 & 0.59164 & 0.11001 & 0.11420 & 0.11514 & 71.66 & 12.61 & 64.33 & -132.62 \\
5 & -0.32852 & 0.60923 & 0.07562 & 0.07889 & 0.07984 & 49.51 & 8.53 & 57.00 & -94.03 \\
6 & -0.32428 & 0.57034 & 0.17854 & 0.18505 & 0.18599 & 116.12 & 21.05 & 78.67 & -209.78 \\
7 & -0.32461 & 0.58156 & 0.14431 & 0.14963 & 0.15057 & 93.89 & 16.82 & 71.51 & -171.20 \\
8 & -0.31806 & 0.56324 & 0.17701 & 0.18336 & 0.18431 & 115.06 & 22.64 & 75.99 & -209.78 \\
9 & -0.27614 & 0.54183 & 0.24560 & 0.25447 & 0.25541 & 159.68 & 30.07 & 91.85 & -286.93 \\
10 & -0.28946 & 0.57924 & 0.14335 & 0.14852 & 0.14947 & 93.20 & 17.07 & 70.14 & -171.19 \\
11 & -0.27438 & 0.53076 & 0.34813 & 0.36077 & 0.36172 & 226.39 & 42.84 & 112.82 & -402.67 \\
\hline
\end{tabular}

Table (10): Physico-chemical parameters in ion phase using HF/STO-3G

\begin{tabular}{llllllllll}
\hline $\begin{array}{l}\text { HF/STO-3G } \\
\text { Ion Phase }\end{array}$ & HOMO & LUMO & $\begin{array}{l}\text { Zero } \\
\text { energy }\end{array}$ & $\begin{array}{l}\text { Thermal } \\
\text { energy }\end{array}$ & $\begin{array}{l}\text { Thermal } \\
\text { enthalpy }\end{array}$ & $\begin{array}{l}\text { Total } \\
\text { energy }\end{array}$ & C.V & $S$ & HF \\
\hline 1 & -0.42665 & 0.09345 & 0.18513 & 0.19183 & 0.19278 & 120.38 & 24.26 & 82.72 & -321.22 \\
2 & -0.74374 & 0.23824 & 0.12480 & 0.12913 & 0.13007 & 81.03 & 12.98 & 65.18 & -133.05 \\
3 & -0.56519 & 0.24793 & 0.32975 & 0.34142 & 0.34236 & 214.24 & 38.60 & 108.44 & -364.55 \\
4 & -0.68432 & 0.23226 & 0.12529 & 0.12962 & 0.13056 & 81.34 & 13.17 & 65.22 & -133.05 \\
5 & -0.77496 & 0.22763 & 0.09104 & 0.09441 & 0.09536 & 59.25 & 8.98 & 57.93 & -94.46 \\
6 & -0.58310 & 0.23587 & 0.19381 & 0.20049 & 0.20144 & 125.81 & 21.66 & 79.64 & -210.21 \\
7 & -0.62493 & 0.23500 & 0.15959 & 0.16508 & 0.16602 & 103.59 & 17.42 & 72.47 & -171.63 \\
8 & -0.63692 & 0.23264 & 0.19208 & 0.19867 & 0.19961 & 124.67 & 23.41 & 77.13 & -210.22 \\
9 & -0.64370 & 0.24623 & 0.26056 & 0.26949 & 0.27043 & 169.10 & 30.46 & 92.85 & -287.39 \\
10 & -0.72427 & 0.24601 & 0.15803 & 0.16337 & 0.16432 & 102.52 & 17.61 & 71.17 & -171.64 \\
11 & -0.59392 & 0.25021 & 0.36318 & 0.37591 & 0.37685 & 235.89 & 43.25 & 113.59 & -403.13 \\
\hline & & & & & & & & &
\end{tabular}

Table (11): Physico-chemical parameters in gas-solvation using HF/STO-3G

\begin{tabular}{llllllllll}
\hline $\begin{array}{l}\text { HF/STO-3G } \\
\text { Gas-Solvation }\end{array}$ & HOMO & LUMO & $\begin{array}{l}\text { Zero } \\
\text { energy }\end{array}$ & $\begin{array}{l}\text { Thermal } \\
\text { energy }\end{array}$ & $\begin{array}{l}\text { Thermal } \\
\text { enthalpy }\end{array}$ & $\begin{array}{l}\text { Total } \\
\text { energy }\end{array}$ & C.V & S & HF \\
\hline 1 & -0.27336 & 0.26783 & 0.16928 & 0.17498 & 0.17593 & 109.80 & 21.64 & 77.32 & -320.79 \\
2 & -0.30956 & 0.59673 & 0.10961 & 0.11379 & 0.11473 & 71.40 & 12.41 & 64.20 & -132.60 \\
3 & -0.30354 & 0.54241 & 0.31428 & 0.32580 & 0.32675 & 204.44 & 37.96 & 107.83 & -364.10 \\
4 & -0.32535 & 0.59898 & 0.10979 & 0.11397 & 0.11491 & 71.52 & 12.59 & 64.31 & -132.62 \\
5 & -0.32899 & 0.61897 & 0.07544 & 0.07872 & 0.07966 & 49.40 & 8.52 & 57.03 & -94.04 \\
6 & -0.32464 & 0.57530 & 0.17822 & 0.18474 & 0.18568 & 115.92 & 21.05 & 78.71 & -209.78 \\
7 & -0.32494 & 0.58764 & 0.14403 & 0.14935 & 0.15030 & 93.72 & 16.81 & 71.54 & -171.20 \\
8 & -0.31838 & 0.56789 & 0.17685 & 0.18317 & 0.18411 & 114.94 & 22.57 & 75.87 & -209.78 \\
9 & -0.27976 & 0.54215 & 0.24541 & 0.25427 & 0.25522 & 159.56 & 30.07 & 92.13 & -286.93 \\
10 & -0.29515 & 0.57791 & 0.14329 & 0.14844 & 0.14939 & 93.15 & 17.04 & 70.06 & -171.19 \\
11 & -0.27923 & 0.53057 & 0.34784 & 0.36047 & 0.36142 & 226.20 & 42.83 & 112.86 & -402.67 \\
\hline
\end{tabular}

Table (12): Physico-chemical parameters in ion-solvation using HF/STO-3G

\begin{tabular}{llllllllll}
\hline $\begin{array}{l}\text { HF/STO-3G } \\
\text { Ion-Solvation }\end{array}$ & HOMO & LUMO & $\begin{array}{l}\text { Zero } \\
\text { energy }\end{array}$ & $\begin{array}{l}\text { Thermal } \\
\text { energy }\end{array}$ & $\begin{array}{l}\text { Thermal } \\
\text { enthalpy }\end{array}$ & $\begin{array}{l}\text { Total } \\
\text { energy }\end{array}$ & C.V & S & HF \\
\hline 1 & -0.29743 & 0.23435 & 0.18391 & 0.19065 & 0.19159 & 119.63 & 24.16 & 84.22 & -321.33 \\
2 & -0.56460 & 0.43052 & 0.12403 & 0.12834 & 0.12928 & 80.53 & 12.89 & 65.06 & -133.15 \\
3 & -0.43638 & 0.42198 & 0.32875 & 0.33954 & 0.34049 & 213.07 & 36.50 & 103.08 & -364.64 \\
4 & -0.51280 & 0.43911 & 0.12443 & 0.12873 & 0.12967 & 80.78 & 13.07 & 65.07 & -133.15 \\
5 & -0.58225 & 0.44172 & 0.09008 & 0.09346 & 0.09440 & 58.65 & 8.94 & 57.91 & -94.57 \\
6 & -0.45316 & 0.43799 & 0.19288 & 0.19954 & 0.20048 & 125.21 & 21.55 & 79.57 & -210.31 \\
7 & -0.47641 & 0.43919 & 0.15869 & 0.16414 & 0.16509 & 103.00 & 17.31 & 72.32 & -171.74 \\
8 & -0.47824 & 0.41958 & 0.19152 & 0.19801 & 0.19895 & 124.25 & 23.13 & 76.66 & -210.32 \\
9 & -0.49569 & 0.40531 & 0.26006 & 0.26804 & 0.26898 & 168.20 & 28.37 & 86.38 & -287.47 \\
10 & -0.55728 & 0.41885 & 0.15752 & 0.16280 & 0.16375 & 102.16 & 17.47 & 70.87 & -171.73 \\
11 & -0.45918 & 0.40465 & 0.36256 & 0.37525 & 0.37620 & 235.48 & 43.14 & 113.67 & -403.22 \\
\hline
\end{tabular}


Table (13): Physico-chemical parameters in gas phase using HF/6-31G

\begin{tabular}{llllllllll}
\hline $\begin{array}{l}\text { HF/6-31G } \\
\text { Gas Phase }\end{array}$ & HOMO & LUMO & $\begin{array}{l}\text { Zero } \\
\text { energy }\end{array}$ & $\begin{array}{l}\text { Thermal } \\
\text { energy }\end{array}$ & $\begin{array}{l}\text { Thermal } \\
\text { enthalpy }\end{array}$ & $\begin{array}{l}\text { Total } \\
\text { energy }\end{array}$ & C.V & S & HF \\
\hline 1 & -0.31859 & 0.15033 & 0.15790 & 0.16458 & 0.16553 & 103.28 & 24.99 & 81.99 & -324.64 \\
2 & -0.34376 & 0.23112 & 0.09904 & 0.10345 & 0.10440 & 64.92 & 13.45 & 64.98 & -134.18 \\
3 & -0.34212 & 0.22905 & 0.28262 & 0.29447 & 0.29542 & 184.79 & 40.25 & 108.77 & -368.30 \\
4 & -0.36164 & 0.23053 & 0.09931 & 0.10368 & 0.10462 & 65.06 & 13.78 & 64.80 & -134.19 \\
5 & -0.36211 & 0.23334 & 0.06840 & 0.07189 & 0.07284 & 45.11 & 9.60 & 57.67 & -95.17 \\
6 & -0.36067 & 0.23028 & 0.16058 & 0.16737 & 0.16832 & 105.03 & 22.77 & 79.50 & -212.23 \\
7 & -0.36099 & 0.23164 & 0.12995 & 0.13551 & 0.13645 & 85.03 & 18.30 & 72.18 & -173.21 \\
8 & -0.36133 & 0.20876 & 0.15916 & 0.16563 & 0.16657 & 103.93 & 24.09 & 76.10 & -212.24 \\
9 & -0.32480 & 0.22592 & 0.22113 & 0.23008 & 0.23102 & 144.38 & 31.43 & 91.07 & -290.24 \\
10 & -0.33212 & 0.22260 & 0.12913 & 0.13452 & 0.13546 & 84.41 & 17.88 & 70.96 & -173.19 \\
11 & -0.32331 & 0.22139 & 0.31267 & 0.32559 & 0.32653 & 204.31 & 45.07 & 112.90 & -407.30 \\
\hline
\end{tabular}

Table (14): Physico-chemical parameters in ion phase using HF/6-31G

\begin{tabular}{llllllllll}
\hline $\begin{array}{l}\text { HF/6-31G } \\
\text { Ion Phase }\end{array}$ & HOMO & LUMO & $\begin{array}{l}\text { Zero } \\
\text { energy }\end{array}$ & $\begin{array}{l}\text { Thermal } \\
\text { energy }\end{array}$ & $\begin{array}{l}\text { Thermal } \\
\text { enthalpy }\end{array}$ & $\begin{array}{l}\text { Total } \\
\text { energy }\end{array}$ & C.V & $S$ & HF \\
\hline 1 & -0.47546 & -0.03050 & 0.17434 & 0.18111 & 0.18205 & 113.65 & 25.02 & 82.52 & -325.02 \\
2 & -0.75429 & -0.02090 & 0.11600 & 0.12040 & 0.12135 & 75.55 & 13.47 & 65.39 & -134.56 \\
3 & -0.59555 & 0.00754 & 0.29944 & 0.31139 & 0.31234 & 195.40 & 40.53 & 109.46 & -368.69 \\
4 & -0.69953 & -0.02884 & 0.11611 & 0.12048 & 0.12143 & 75.61 & 13.67 & 65.23 & -134.57 \\
5 & -0.78799 & -0.04068 & 0.08547 & 0.08889 & 0.08984 & 55.78 & 9.25 & 58.00 & -95.54 \\
6 & -0.60833 & -0.02357 & 0.17746 & 0.18428 & 0.18523 & 115.64 & 22.68 & 80.01 & -212.61 \\
7 & -0.64583 & -0.02538 & 0.14683 & 0.15241 & 0.15336 & 95.64 & 18.19 & 72.66 & -173.59 \\
8 & -0.66218 & -0.01551 & 0.17565 & 0.18224 & 0.18318 & 114.36 & 24.29 & 76.78 & -212.62 \\
9 & -0.66024 & 0.03149 & 0.23788 & 0.24690 & 0.24784 & 154.93 & 31.85 & 92.23 & -290.64 \\
10 & -0.73598 & -0.00360 & 0.14592 & 0.15134 & 0.15229 & 94.97 & 18.28 & 71.37 & -173.58 \\
11 & -0.61758 & 0.04044 & 0.32972 & 0.34270 & 0.34365 & 215.05 & 45.42 & 113.78 & -407.70 \\
\hline
\end{tabular}

Table (15): Physico-chemical parameters in gas-solvation using HF/6-31G

\begin{tabular}{llllllllll}
\hline $\begin{array}{l}\text { HF/6-31G } \\
\text { Gas-Solvation }\end{array}$ & HOMO & LUMO & $\begin{array}{l}\text { Zero } \\
\text { energy }\end{array}$ & $\begin{array}{l}\text { Thermal } \\
\text { energy }\end{array}$ & $\begin{array}{l}\text { Thermal } \\
\text { enthalpy }\end{array}$ & $\begin{array}{l}\text { Total } \\
\text { energy }\end{array}$ & C.V & S & HF \\
\hline 1 & -0.32405 & 0.14400 & 0.15648 & 0.16237 & 0.16332 & 101.89 & 22.95 & 77.82 & -324.65 \\
2 & -0.35088 & 0.23499 & 0.09872 & 0.10307 & 0.10401 & 64.68 & 13.27 & 64.74 & -134.19 \\
3 & -0.34827 & 0.23137 & 0.28162 & 0.29349 & 0.29443 & 184.17 & 40.21 & 109.55 & -368.30 \\
4 & -0.36750 & 0.23673 & 0.09883 & 0.10314 & 0.10408 & 64.72 & 13.54 & 64.61 & -134.20 \\
5 & -0.36914 & 0.24045 & 0.06805 & 0.07148 & 0.07242 & 44.85 & 9.30 & 57.47 & -95.18 \\
6 & -0.36682 & 0.23398 & 0.15991 & 0.16665 & 0.16759 & 104.57 & 22.55 & 79.38 & -212.24 \\
7 & -0.36718 & 0.23646 & 0.12938 & 0.13488 & 0.13582 & 84.64 & 18.06 & 72.03 & -173.22 \\
8 & -0.36563 & 0.21376 & 0.15873 & 0.16513 & 0.16607 & 103.62 & 23.83 & 75.83 & -212.24 \\
9 & -0.32766 & 0.22828 & 0.22068 & 0.22961 & 0.23055 & 144.08 & 31.47 & 90.96 & -290.25 \\
10 & -0.34022 & 0.22339 & 0.12898 & 0.13432 & 0.13526 & 84.28 & 17.86 & 70.71 & -173.19 \\
11 & -0.32921 & 0.22373 & 0.31205 & 0.32494 & 0.32589 & 203.90 & 45.09 & 112.69 & -407.30 \\
\hline
\end{tabular}

Table (16): Physico-chemical parameters in ion-solvation using HF/6-31G

\begin{tabular}{llllllllll}
\hline $\begin{array}{l}\text { HF/6-31G } \\
\text { Ion-Solvation }\end{array}$ & HOMO & LUMO & $\begin{array}{l}\text { Zero } \\
\text { energy }\end{array}$ & $\begin{array}{l}\text { Thermal } \\
\text { energy }\end{array}$ & $\begin{array}{l}\text { Thermal } \\
\text { enthalpy }\end{array}$ & $\begin{array}{l}\text { Total } \\
\text { energy }\end{array}$ & C.V & $S$ & HF \\
\hline 1 & -0.34694 & 0.11843 & 0.17218 & 0.17907 & 0.18002 & 112.37 & 24.93 & 87.11 & -325.12 \\
2 & -0.57686 & 0.17430 & 0.11475 & 0.11913 & 0.12007 & 74.75 & 13.38 & 65.26 & -134.66 \\
3 & -0.46780 & 0.18722 & 0.29743 & 0.30858 & 0.30952 & 193.63 & 38.51 & 104.84 & -368.78 \\
4 & -0.52703 & 0.17835 & 0.11475 & 0.11910 & 0.12004 & 74.73 & 13.56 & 65.11 & -134.67 \\
5 & -0.59475 & 0.17382 & 0.08413 & 0.08754 & 0.08849 & 54.93 & 9.18 & 57.96 & -95.65 \\
6 & -0.47680 & 0.18086 & 0.17585 & 0.18268 & 0.18362 & 114.63 & 22.58 & 80.19 & -212.71 \\
7 & -0.49430 & 0.18061 & 0.14532 & 0.15089 & 0.15183 & 94.68 & 18.08 & 72.66 & -173.69 \\
8 & -0.50357 & 0.16895 & 0.17456 & 0.18105 & 0.18199 & 113.61 & 23.97 & 76.35 & -212.72 \\
9 & -0.51386 & 0.18875 & 0.23670 & 0.24578 & 0.24672 & 154.23 & 31.84 & 97.76 & -290.72 \\
10 & -0.57107 & 0.17087 & 0.14499 & 0.15036 & 0.15131 & 94.35 & 18.17 & 71.08 & -173.67 \\
11 & -0.48414 & 0.19675 & 0.32817 & 0.34115 & 0.34210 & 214.08 & 45.38 & 114.16 & -407.78 \\
\hline
\end{tabular}


Table (17): Physico-chemical parameters in gas phase using HF/6-31G(d,p)

\begin{tabular}{llllllllll}
\hline $\begin{array}{l}\text { HF/6-31G(d,p) } \\
\text { Gas Phase }\end{array}$ & HOMO & LUMO & $\begin{array}{l}\text { Zero } \\
\text { energy }\end{array}$ & $\begin{array}{l}\text { Thermal } \\
\text { energy }\end{array}$ & $\begin{array}{l}\text { Thermal } \\
\text { enthalpy }\end{array}$ & $\begin{array}{l}\text { Total } \\
\text { energy }\end{array}$ & C.V & S & HF \\
\hline 1 & -0.31807 & 0.14950 & 0.15645 & 0.16316 & 0.16410 & 102.38 & 25.11 & 82.23 & -324.78 \\
2 & -0.36063 & 0.23017 & 0.09880 & 0.10306 & 0.10400 & 64.67 & 13.06 & 64.33 & -134.25 \\
3 & -0.35751 & 0.22848 & 0.28066 & 0.29246 & 0.29340 & 183.52 & 40.44 & 108.07 & -368.48 \\
4 & -0.38102 & 0.22767 & 0.09907 & 0.10331 & 0.10425 & 64.83 & 13.36 & 64.27 & -134.26 \\
5 & -0.38256 & 0.22992 & 0.06849 & 0.07183 & 0.07277 & 45.07 & 9.03 & 57.11 & -95.22 \\
6 & -0.38001 & 0.22885 & 0.15986 & 0.16652 & 0.16747 & 104.50 & 22.47 & 78.94 & -212.34 \\
7 & -0.38042 & 0.22951 & 0.12947 & 0.13490 & 0.13585 & 84.65 & 17.94 & 71.63 & -173.30 \\
8 & -0.37854 & 0.20750 & 0.15847 & 0.16485 & 0.16579 & 103.44 & 23.81 & 75.77 & -212.34 \\
9 & -0.33738 & 0.22783 & 0.21955 & 0.22845 & 0.22939 & 143.35 & 31.60 & 90.81 & -290.39 \\
10 & -0.34581 & 0.22409 & 0.12857 & 0.13380 & 0.13475 & 83.96 & 17.68 & 67.99 & -173.28 \\
11 & -0.33580 & 0.22324 & 0.31038 & 0.32326 & 0.32420 & 202.85 & 45.43 & 112.45 & -407.51 \\
\hline
\end{tabular}

Table (18): Physico-chemical parameters in ion phase using HF/6-31G(d,p)

\begin{tabular}{llllllllll}
\hline $\begin{array}{l}\text { HF/6-31G(d,p) } \\
\text { Ion Phase }\end{array}$ & HOMO & LUMO & $\begin{array}{l}\text { Zero } \\
\text { energy }\end{array}$ & $\begin{array}{l}\text { Thermal } \\
\text { energy }\end{array}$ & $\begin{array}{l}\text { Thermal } \\
\text { enthalpy }\end{array}$ & $\begin{array}{l}\text { Total } \\
\text { energy }\end{array}$ & C.V & S & HF \\
\hline 1 & -0.47274 & -0.02871 & 0.17217 & 0.17904 & 0.17998 & 112.35 & 25.62 & 82.78 & -325.15 \\
2 & -0.75589 & -0.01977 & 0.11520 & 0.11956 & 0.12050 & 75.03 & 13.50 & 65.10 & -134.63 \\
3 & -0.59858 & 0.00943 & 0.29712 & 0.30909 & 0.31003 & 193.96 & 41.00 & 109.16 & -368.88 \\
4 & -0.70088 & -0.02809 & 0.11503 & 0.11939 & 0.12034 & 74.92 & 13.81 & 65.11 & -134.63 \\
5 & -0.78687 & -0.03986 & 0.08465 & 0.08804 & 0.08899 & 55.25 & 9.30 & 57.85 & -95.59 \\
6 & -0.61044 & -0.02257 & 0.17592 & 0.18274 & 0.18369 & 114.67 & 22.93 & 79.86 & -212.71 \\
7 & -0.64784 & -0.02442 & 0.14552 & 0.15110 & 0.15205 & 94.82 & 18.38 & 72.52 & -173.67 \\
8 & -0.66338 & -0.01513 & 0.17419 & 0.18077 & 0.18172 & 113.44 & 24.45 & 76.72 & -212.72 \\
9 & -0.66336 & 0.03232 & 0.23609 & 0.24512 & 0.24607 & 153.82 & 32.11 & 92.41 & -290.79 \\
10 & -0.73994 & -0.00253 & 0.14514 & 0.15050 & 0.15145 & 94.44 & 18.24 & 70.99 & -173.66 \\
11 & -0.62033 & 0.04193 & 0.32725 & 0.34025 & 0.34120 & 213.51 & 45.87 & 113.66 & -407.90 \\
\hline
\end{tabular}

Table (19): Physico-chemical parameters in gas-solvation using HF/6-31G(d,p)

\begin{tabular}{llllllllll}
\hline $\begin{array}{l}\text { HF/6-31G(d,p) } \\
\text { Gas-Solvation }\end{array}$ & HOMO & LUMO & $\begin{array}{l}\text { Zero } \\
\text { energy }\end{array}$ & $\begin{array}{l}\text { Thermal } \\
\text { energy }\end{array}$ & $\begin{array}{l}\text { Thermal } \\
\text { enthalpy }\end{array}$ & $\begin{array}{l}\text { Total } \\
\text { energy }\end{array}$ & C.V & S & HF \\
\hline 1 & -0.32232 & 0.14432 & 0.15492 & 0.16086 & 0.16180 & 100.94 & 23.25 & 77.93 & -324.79 \\
2 & -0.36398 & 0.23557 & 0.09838 & 0.10263 & 0.10357 & 64.40 & 13.03 & 64.29 & -134.26 \\
3 & -0.36056 & 0.23256 & 0.27950 & 0.29137 & 0.29231 & 182.84 & 40.52 & 109.47 & -368.49 \\
4 & -0.38246 & 0.23643 & 0.09841 & 0.10265 & 0.10360 & 64.42 & 13.35 & 64.30 & -134.27 \\
5 & -0.38501 & 0.23992 & 0.06796 & 0.07130 & 0.07224 & 44.74 & 9.00 & 57.14 & -95.20 \\
6 & -0.38144 & 0.23523 & 0.15897 & 0.16566 & 0.16660 & 103.95 & 22.50 & 79.11 & -212.35 \\
7 & -0.38186 & 0.23709 & 0.12869 & 0.13414 & 0.13508 & 84.17 & 17.95 & 71.75 & -173.31 \\
8 & -0.37924 & 0.21437 & 0.15789 & 0.16425 & 0.16519 & 103.07 & 23.74 & 75.65 & -212.35 \\
9 & -0.34010 & 0.23012 & 0.21907 & 0.22797 & 0.22892 & 143.06 & 31.64 & 90.93 & -290.40 \\
10 & -0.35164 & 0.22556 & 0.12841 & 0.13361 & 0.13456 & 83.84 & 17.66 & 70.03 & -173.29 \\
11 & -0.33977 & 0.22594 & 0.30968 & 0.32256 & 0.32350 & 202.41 & 45.47 & 112.51 & -407.51 \\
\hline
\end{tabular}

Table (20): Physico-chemical parameters in ion-solvation using HF/6-31G(d,p)

\begin{tabular}{llllllllll}
\hline $\begin{array}{l}\text { HF/6-31G(d,p) } \\
\text { Ion Solvation }\end{array}$ & HOMO & LUMO & $\begin{array}{l}\text { Zero } \\
\text { energy }\end{array}$ & $\begin{array}{l}\text { Thermal } \\
\text { energy }\end{array}$ & $\begin{array}{l}\text { Thermal } \\
\text { enthalpy }\end{array}$ & $\begin{array}{l}\text { Total } \\
\text { energy }\end{array}$ & C.V & S & HF \\
\hline 1 & -0.34359 & 0.12017 & 0.16995 & 0.17601 & 0.17696 & 110.45 & 23.57 & 78.69 & -325.26 \\
2 & -0.57782 & 0.17587 & 0.11394 & 0.11828 & 0.11922 & 74.22 & 13.41 & 64.99 & -134.73 \\
3 & -0.47080 & 0.18946 & 0.29506 & 0.30621 & 0.30715 & 192.15 & 38.98 & 104.48 & -368.97 \\
4 & -0.52804 & 0.17961 & 0.11365 & 0.11799 & 0.11894 & 74.04 & 13.70 & 64.98 & -134.74 \\
5 & -0.59351 & 0.17506 & 0.08329 & 0.08668 & 0.08763 & 54.39 & 9.25 & 57.82 & -95.70 \\
6 & -0.47887 & 0.18237 & 0.17427 & 0.18110 & 0.18204 & 113.64 & 22.85 & 80.02 & -212.82 \\
7 & -0.49615 & 0.18211 & 0.14399 & 0.14955 & 0.15050 & 93.85 & 18.28 & 72.50 & -173.78 \\
8 & -0.50484 & 0.17040 & 0.17306 & 0.17955 & 0.18049 & 112.67 & 24.16 & 76.32 & -212.82 \\
9 & -0.51675 & 0.19014 & 0.23493 & 0.24307 & 0.24402 & 152.53 & 30.11 & 86.58 & -290.87 \\
10 & -0.57435 & 0.17242 & 0.14426 & 0.14956 & 0.15051 & 93.85 & 18.11 & 70.72 & -173.76 \\
11 & -0.48663 & 0.19904 & 0.32565 & 0.33869 & 0.33963 & 212.53 & 45.83 & 114.99 & -407.99 \\
\hline
\end{tabular}


Table (21): Physico-chemical parameters in gas phase using DFT/STO-3G

\begin{tabular}{llllllllll}
\hline $\begin{array}{l}\text { DFT/STO-3G } \\
\text { Gas Phase }\end{array}$ & HOMO & LUMO & $\begin{array}{l}\text { Zero } \\
\text { energy }\end{array}$ & $\begin{array}{l}\text { Thermal } \\
\text { energy }\end{array}$ & $\begin{array}{l}\text { Thermal } \\
\text { enthalpy }\end{array}$ & $\begin{array}{l}\text { Total } \\
\text { energy }\end{array}$ & C.V & S & HF \\
\hline 1 & -0.15302 & 0.09195 & 0.15518 & 0.16219 & 0.16313 & 101.77 & 26.12 & 83.74 & -322.86 \\
2 & -0.13665 & 0.32111 & 0.10034 & 0.10464 & 0.10559 & 65.67 & 13.27 & 64.67 & -133.49 \\
3 & -0.13082 & 0.29280 & 0.28842 & 0.30050 & 0.30144 & 188.57 & 40.84 & 109.74 & -366.58 \\
4 & -0.14530 & 0.32717 & 0.10050 & 0.10486 & 0.10580 & 65.80 & 13.49 & 65.05 & -133.49 \\
5 & -0.14786 & 0.33551 & 0.06900 & 0.07233 & 0.07327 & 45.38 & 8.99 & 57.27 & -94.64 \\
6 & -0.14476 & 0.31621 & 0.16341 & 0.17024 & 0.17118 & 106.82 & 22.59 & 80.11 & -211.18 \\
7 & -0.14498 & 0.32145 & 0.13199 & 0.13756 & 0.13850 & 86.32 & 18.03 & 72.59 & -172.34 \\
8 & -0.14191 & 0.31013 & 0.16192 & 0.16875 & 0.16970 & 105.90 & 24.45 & 77.98 & -211.19 \\
9 & -0.11636 & 0.28671 & 0.22483 & 0.23419 & 0.23513 & 146.96 & 32.51 & 93.47 & -288.88 \\
10 & -0.12758 & 0.30833 & 0.13114 & 0.13653 & 0.13747 & 85.67 & 18.31 & 70.91 & -172.33 \\
11 & -0.11428 & 0.28035 & 0.31881 & 0.33224 & 0.33319 & 208.49 & 46.27 & 116.83 & -405.42 \\
\hline
\end{tabular}

Table (22): Physico-chemical parameters in ion phase using DFT/STO-3G

\begin{tabular}{llllllllll}
\hline $\begin{array}{l}\text { DFT/STO-3G } \\
\text { Ion phase }\end{array}$ & HOMO & LUMO & $\begin{array}{l}\text { Zero } \\
\text { energy }\end{array}$ & $\begin{array}{l}\text { Thermal } \\
\text { energy }\end{array}$ & $\begin{array}{l}\text { Thermal } \\
\text { enthalpy }\end{array}$ & $\begin{array}{l}\text { Total } \\
\text { energy }\end{array}$ & C.V & $S$ & HF \\
\hline 1 & -0.33938 & -0.09297 & 0.16962 & 0.17683 & 0.17778 & 110.97 & 26.96 & 84.44 & -323.30 \\
2 & -0.59124 & -0.02841 & 0.11477 & 0.11927 & 0.12022 & 74.84 & 13.94 & 65.90 & -133.92 \\
3 & -0.42618 & -0.00805 & 0.30271 & 0.31498 & 0.31593 & 197.66 & 41.61 & 111.01 & -367.00 \\
4 & -0.53303 & -0.02840 & 0.11520 & 0.11970 & 0.12064 & 75.11 & 14.11 & 65.92 & -133.92 \\
5 & -0.61691 & -0.03649 & 0.08386 & 0.08731 & 0.08825 & 54.79 & 9.51 & 58.25 & -95.06 \\
6 & -0.43600 & -0.02361 & 0.17804 & 0.18505 & 0.18600 & 116.12 & 23.29 & 81.07 & -211.62 \\
7 & -0.47624 & -0.02490 & 0.14662 & 0.15238 & 0.15333 & 95.62 & 18.72 & 73.62 & -172.77 \\
8 & -0.49721 & -0.01973 & 0.17627 & 0.18334 & 0.18428 & 115.05 & 25.27 & 79.07 & -211.63 \\
9 & -0.50045 & -0.01067 & 0.23907 & 0.24854 & 0.24948 & 155.96 & 32.96 & 95.14 & -289.34 \\
10 & -0.57635 & -0.02188 & 0.14519 & 0.15085 & 0.15179 & 94.66 & 19.04 & 72.48 & -172.77 \\
11 & -0.45397 & -0.00423 & 0.33316 & 0.34671 & 0.34765 & 217.56 & 46.77 & 117.77 & -405.89 \\
\hline
\end{tabular}

Table (23): Physico-chemical parameters in gas-solvation using DFT/STO-3G

\begin{tabular}{llllllllll}
\hline $\begin{array}{l}\text { DFT/STO-3G } \\
\text { Gas-Solvation }\end{array}$ & HOMO & LUMO & $\begin{array}{l}\text { Zero } \\
\text { energy }\end{array}$ & $\begin{array}{l}\text { Thermal } \\
\text { energy }\end{array}$ & $\begin{array}{l}\text { Thermal } \\
\text { enthalpy }\end{array}$ & $\begin{array}{l}\text { Total } \\
\text { energy }\end{array}$ & C.V & S & HF \\
\hline 1 & -0.14937 & 0.08881 & 0.15432 & 0.16053 & 0.16147 & 100.73 & 24.20 & 79.11 & -322.87 \\
2 & -0.13900 & 0.32411 & 0.10013 & 0.10443 & 0.10538 & 65.53 & 13.25 & 64.68 & -133.49 \\
3 & -0.13392 & 0.29466 & 0.28780 & 0.29990 & 0.30085 & 188.19 & 40.86 & 110.38 & -366.59 \\
4 & -0.14515 & 0.33500 & 0.10022 & 0.10458 & 0.10552 & 65.62 & 13.48 & 65.05 & -133.49 \\
5 & -0.14782 & 0.34453 & 0.06878 & 0.07211 & 0.07305 & 45.25 & 8.97 & 57.31 & -94.64 \\
6 & -0.14477 & 0.32211 & 0.16298 & 0.16982 & 0.17076 & 106.56 & 22.59 & 80.14 & -211.19 \\
7 & -0.14503 & 0.32832 & 0.13162 & 0.13720 & 0.13815 & 86.10 & 18.04 & 72.67 & -172.34 \\
8 & -0.14223 & 0.31511 & 0.16171 & 0.16851 & 0.16945 & 105.74 & 24.38 & 77.79 & -211.19 \\
9 & -0.12180 & 0.28577 & 0.22462 & 0.23396 & 0.23490 & 146.81 & 32.51 & 93.16 & -288.88 \\
10 & -0.13389 & 0.30642 & 0.13107 & 0.13645 & 0.13739 & 85.62 & 18.30 & 70.84 & -172.33 \\
11 & -0.12088 & 0.27882 & 0.31846 & 0.33185 & 0.33280 & 208.24 & 46.28 & 116.61 & -405.42 \\
\hline
\end{tabular}

Table (24): Physico-chemical parameters in ion-solvation using DFT/STO-3G

\begin{tabular}{|c|c|c|c|c|c|c|c|c|c|}
\hline $\begin{array}{l}\text { DFT/STO-3G } \\
\text { Ion Solvation }\end{array}$ & HOMO & LUMO & $\begin{array}{l}\text { Zero } \\
\text { energy }\end{array}$ & $\begin{array}{l}\text { Thermal } \\
\text { energy }\end{array}$ & $\begin{array}{l}\text { Thermal } \\
\text { enthalpy }\end{array}$ & $\begin{array}{l}\text { Total } \\
\text { energy }\end{array}$ & C.V & $S$ & $\mathrm{HF}$ \\
\hline 1 & -0.20931 & 0.05285 & 0.16829 & 0.17553 & 0.17647 & 110.14 & 26.78 & 85.47 & -323.41 \\
\hline 2 & -0.41289 & 0.16372 & 0.11381 & 0.11828 & 0.11923 & 74.22 & 13.83 & 65.79 & -134.02 \\
\hline 3 & -0.30155 & 0.16670 & 0.30152 & 0.31291 & 0.31385 & 196.35 & 39.48 & 105.63 & -367.12 \\
\hline 4 & -0.36303 & 0.17752 & 0.11420 & 0.11868 & 0.11963 & 74.48 & 14.01 & 65.80 & -134.03 \\
\hline 5 & -0.42574 & 0.17585 & 0.08275 & 0.08620 & 0.08715 & 54.09 & 9.48 & 58.25 & -95.18 \\
\hline 6 & -0.31018 & 0.17803 & 0.17699 & 0.18397 & 0.18492 & 115.44 & 23.15 & 80.95 & -211.73 \\
\hline 7 & -0.32947 & 0.17848 & 0.14560 & 0.15132 & 0.15226 & 94.95 & 18.59 & 73.45 & -172.88 \\
\hline 8 & -0.33930 & 0.16624 & 0.17563 & 0.18257 & 0.18352 & 114.57 & 24.95 & 78.51 & -211.73 \\
\hline 9 & -0.35377 & 0.14764 & 0.23834 & 0.24688 & 0.24782 & 154.92 & 30.86 & 88.73 & -289.42 \\
\hline 10 & -0.40933 & 0.15096 & 0.14443 & 0.15002 & 0.15097 & 94.14 & 18.89 & 72.15 & -172.87 \\
\hline 11 & -0.32183 & 0.15004 & 0.33224 & 0.34575 & 0.34669 & 216.96 & 46.66 & 117.24 & -405.97 \\
\hline
\end{tabular}


Table (25): Physico-chemical parameters in gas phase using DFT/6-31G

\begin{tabular}{llllllllll}
\hline $\begin{array}{l}\text { DFT/6-31G } \\
\text { Gas Phase }\end{array}$ & HOMO & LUMO & $\begin{array}{l}\text { Zero } \\
\text { energy }\end{array}$ & $\begin{array}{l}\text { Thermal } \\
\text { energy }\end{array}$ & $\begin{array}{l}\text { Thermal } \\
\text { enthalpy }\end{array}$ & $\begin{array}{l}\text { Total } \\
\text { energy }\end{array}$ & C.V & S & HF \\
\hline 1 & -0.21450 & 0.00788 & 0.14727 & 0.15442 & 0.15536 & 96.90 & 27.32 & 83.61 & -326.83 \\
2 & -0.19849 & 0.08756 & 0.09294 & 0.09743 & 0.09837 & 61.14 & 14.05 & 65.18 & -135.12 \\
3 & -0.19909 & 0.09047 & 0.26565 & 0.27797 & 0.27891 & 174.43 & 42.67 & 110.53 & -370.95 \\
4 & -0.20743 & 0.08631 & 0.09319 & 0.09770 & 0.09864 & 61.31 & 14.56 & 65.33 & -135.13 \\
5 & -0.20597 & 0.08790 & 0.06413 & 0.06768 & 0.06862 & 42.47 & 10.01 & 57.87 & -95.83 \\
6 & -0.20723 & 0.08782 & 0.15090 & 0.15795 & 0.15889 & 99.11 & 24.10 & 80.50 & -213.74 \\
7 & -0.20742 & 0.08786 & 0.12202 & 0.12779 & 0.12873 & 80.19 & 19.37 & 72.99 & -174.44 \\
8 & -0.21039 & 0.06965 & 0.14943 & 0.15631 & 0.15726 & 98.09 & 25.71 & 77.68 & -213.75 \\
9 & -0.18969 & 0.08656 & 0.20756 & 0.21699 & 0.21793 & 136.16 & 33.49 & 93.53 & -292.34 \\
10 & -0.19464 & 0.08322 & 0.12120 & 0.12672 & 0.12766 & 79.52 & 18.82 & 71.36 & -174.42 \\
11 & -0.18922 & 0.08387 & 0.29370 & 0.30727 & 0.30821 & 192.81 & 47.97 & 116.18 & -410.25 \\
\hline
\end{tabular}

Table (26): Physico-chemical parameters in ion phase using DFT/6-31G

\begin{tabular}{llllllllll}
\hline $\begin{array}{l}\text { DFT/6-31G } \\
\text { Ion Phase }\end{array}$ & HOMO & LUMO & $\begin{array}{l}\text { Zero } \\
\text { energy }\end{array}$ & $\begin{array}{l}\text { Thermal } \\
\text { energy }\end{array}$ & $\begin{array}{l}\text { Thermal } \\
\text { enthalpy }\end{array}$ & $\begin{array}{l}\text { Total } \\
\text { energy }\end{array}$ & C.V & S & HF \\
\hline 1 & -0.39288 & -0.17508 & 0.16273 & 0.16996 & 0.17091 & 106.65 & 27.40 & 84.20 & -327.21 \\
2 & -0.60383 & -0.16981 & 0.10909 & 0.11360 & 0.11454 & 71.28 & 14.20 & 65.76 & -135.50 \\
3 & -0.45670 & -0.13854 & 0.28161 & 0.29401 & 0.29496 & 184.50 & 43.07 & 111.02 & -371.30 \\
4 & -0.55143 & -0.17609 & 0.10910 & 0.11361 & 0.11455 & 71.29 & 14.44 & 65.72 & -135.50 \\
5 & -0.62917 & -0.18886 & 0.08035 & 0.08382 & 0.08476 & 52.60 & 9.65 & 58.16 & -96.19 \\
6 & -0.46623 & -0.17079 & 0.16682 & 0.17389 & 0.17484 & 109.12 & 24.03 & 81.01 & -214.12 \\
7 & -0.50196 & -0.17274 & 0.13800 & 0.14379 & 0.14473 & 90.23 & 19.25 & 73.46 & -174.81 \\
8 & -0.52475 & -0.15995 & 0.16492 & 0.17191 & 0.17285 & 107.88 & 25.90 & 78.38 & -214.13 \\
9 & -0.51942 & -0.11575 & 0.22347 & 0.23294 & 0.23388 & 146.17 & 33.96 & 94.46 & -292.73 \\
10 & -0.59147 & -0.15222 & 0.13723 & 0.14286 & 0.14380 & 89.64 & 19.41 & 72.15 & -174.80 \\
11 & -0.47967 & -0.10821 & 0.30986 & 0.32348 & 0.32443 & 202.99 & 48.40 & 116.67 & -410.65 \\
\hline
\end{tabular}

Table (27): Physico-chemical parameters in gas-solvation using DFT/6-31G

\begin{tabular}{llllllllll}
\hline $\begin{array}{l}\text { DFT/6-31G } \\
\text { Gas-Solvation }\end{array}$ & HOMO & LUMO & $\begin{array}{l}\text { Zero } \\
\text { energy }\end{array}$ & $\begin{array}{l}\text { Thermal } \\
\text { energy }\end{array}$ & $\begin{array}{l}\text { Thermal } \\
\text { enthalpy }\end{array}$ & $\begin{array}{l}\text { Total } \\
\text { energy }\end{array}$ & C.V & S & HF \\
\hline 1 & -0.21509 & 0.00428 & 0.14594 & 0.15315 & 0.15409 & 96.10 & 27.26 & 85.22 & -326.83 \\
2 & -0.20369 & 0.09324 & 0.09256 & 0.09699 & 0.09794 & 60.86 & 13.93 & 65.01 & -135.12 \\
3 & -0.20422 & 0.09385 & 0.26460 & 0.27695 & 0.27790 & 173.79 & 42.69 & 111.87 & -370.96 \\
4 & -0.21233 & 0.09429 & 0.09267 & 0.09712 & 0.09806 & 60.94 & 14.32 & 65.12 & -135.13 \\
5 & -0.21207 & 0.09659 & 0.06372 & 0.06720 & 0.06814 & 42.17 & 9.71 & 57.65 & -95.83 \\
6 & -0.21233 & 0.09505 & 0.15018 & 0.15718 & 0.15812 & 98.63 & 23.89 & 80.41 & -213.74 \\
7 & -0.21276 & 0.09640 & 0.12141 & 0.12712 & 0.12807 & 79.77 & 19.11 & 72.88 & -174.44 \\
8 & -0.21406 & 0.07563 & 0.14893 & 0.15575 & 0.15669 & 97.73 & 25.49 & 77.47 & -213.75 \\
9 & -0.19418 & 0.08900 & 0.20726 & 0.21662 & 0.21757 & 135.93 & 33.49 & 93.09 & -292.33 \\
10 & -0.20168 & 0.08396 & 0.12110 & 0.12657 & 0.12751 & 79.42 & 18.81 & 71.12 & -174.42 \\
11 & -0.19449 & 0.08611 & 0.29319 & 0.30669 & 0.30764 & 192.45 & 47.98 & 115.49 & -410.25 \\
\hline
\end{tabular}

Table (28): Physico-chemical parameters in ion-solvation using DFT/6-31G

\begin{tabular}{llllllllll}
\hline $\begin{array}{l}\text { DFT/6-31G } \\
\text { Ion Solvation }\end{array}$ & HOMO & LUMO & $\begin{array}{l}\text { Zero } \\
\text { energy }\end{array}$ & $\begin{array}{l}\text { Thermal } \\
\text { energy }\end{array}$ & $\begin{array}{l}\text { Thermal } \\
\text { enthalpy }\end{array}$ & $\begin{array}{l}\text { Total } \\
\text { energy }\end{array}$ & C.V & S & HF \\
\hline 1 & -0.26196 & -0.02471 & 0.16068 & 0.16709 & 0.16804 & 104.85 & 25.28 & 80.01 & -327.31 \\
2 & -0.42716 & 0.02733 & 0.10777 & 0.11225 & 0.11320 & 70.44 & 14.11 & 65.65 & -135.60 \\
3 & -0.33515 & 0.04173 & 0.27962 & 0.29120 & 0.29214 & 182.73 & 41.00 & 106.29 & -371.43 \\
4 & -0.38001 & 0.03285 & 0.10774 & 0.11222 & 0.11316 & 70.42 & 14.32 & 65.60 & -135.61 \\
5 & -0.43786 & 0.02718 & 0.07894 & 0.08240 & 0.08335 & 51.71 & 9.60 & 58.13 & -96.30 \\
6 & -0.33608 & 0.03439 & 0.16525 & 0.17231 & 0.17326 & 108.13 & 23.91 & 81.11 & -214.22 \\
7 & -0.35163 & 0.03418 & 0.13650 & 0.14226 & 0.14320 & 89.27 & 19.13 & 73.43 & -174.91 \\
8 & -0.36705 & 0.02765 & 0.16384 & 0.17072 & 0.17167 & 107.13 & 25.57 & 77.91 & -214.22 \\
9 & -0.37015 & 0.04313 & 0.22249 & 0.23185 & 0.23280 & 145.49 & 33.90 & 92.60 & -292.81 \\
10 & -0.42663 & 0.02346 & 0.13619 & 0.14177 & 0.14271 & 88.96 & 19.29 & 71.88 & -174.89 \\
11 & -0.34806 & 0.04746 & 0.30819 & 0.32188 & 0.32282 & 201.98 & 48.40 & 118.17 & -410.72 \\
\hline
\end{tabular}

Table (29): Physico-chemical parameters in gas phase using DFT/6-31G(d,p)

\begin{tabular}{llllllllll}
\hline $\begin{array}{l}\text { DFT/6-31G(d,p) } \\
\text { Gas Phase }\end{array}$ & HOMO & LUMO & $\begin{array}{l}\text { Zero } \\
\text { energy }\end{array}$ & $\begin{array}{l}\text { Thermal } \\
\text { energy }\end{array}$ & $\begin{array}{l}\text { Thermal } \\
\text { enthalpy }\end{array}$ & $\begin{array}{l}\text { Total } \\
\text { energy }\end{array}$ & C.V & S & HF \\
\hline 1 & -0.22939 & 0.00519 & 0.14628 & 0.15341 & 0.15435 & 96.27 & 27.29 & 83.74 & -326.92 \\
2 & -0.21507 & 0.08720 & 0.09268 & 0.09705 & 0.09799 & 60.90 & 13.79 & 64.70 & -135.17 \\
3 & -0.21407 & 0.08891 & 0.26386 & 0.27614 & 0.27708 & 173.28 & 42.98 & 109.81 & -371.07 \\
4 & -0.22913 & 0.08415 & 0.09298 & 0.09736 & 0.09831 & 61.10 & 14.16 & 64.83 & -135.18 \\
5 & -0.22894 & 0.08544 & 0.06420 & 0.06759 & 0.06854 & 42.42 & 9.46 & 57.34 & -95.86 \\
\hline
\end{tabular}




\begin{tabular}{|c|c|c|c|c|c|c|c|c|c|}
\hline 6 & -0.22871 & 0.08591 & 0.15021 & 0.15714 & 0.15808 & 98.61 & 23.84 & 80.00 & -213.81 \\
\hline 7 & -0.22896 & 0.08593 & 0.12158 & 0.12723 & 0.12817 & 79.84 & 19.03 & 72.49 & -174.49 \\
\hline 8 & -0.22982 & 0.06876 & 0.14868 & 0.15550 & 0.15644 & 97.58 & 25.51 & 77.43 & -213.82 \\
\hline 9 & -0.20105 & 0.08751 & 0.20608 & 0.21548 & 0.21643 & 135.22 & 33.74 & 93.64 & -292.43 \\
\hline 11 & -0.20020 & 0.08479 & 0.29158 & 0.30511 & 0.30605 & 191.46 & 48.42 & 115.38 & -410.38 \\
\hline
\end{tabular}

Table (30): Physico-chemical parameters in ion phase using DFT/6-31G(d,p)

\begin{tabular}{|c|c|c|c|c|c|c|c|c|c|}
\hline $\begin{array}{l}\text { DFT/6-31G(d,p) } \\
\text { Ion Phase }\end{array}$ & HOMO & LUMO & $\begin{array}{l}\text { Zero } \\
\text { energy }\end{array}$ & $\begin{array}{l}\text { Thermal } \\
\text { energy }\end{array}$ & $\begin{array}{l}\text { Thermal } \\
\text { enthalpy }\end{array}$ & $\begin{array}{l}\text { Total } \\
\text { energy }\end{array}$ & C.V & $\mathrm{S}$ & $\mathrm{HF}$ \\
\hline 2 & -0.60315 & -0.16785 & 0.10821 & 0.11270 & 0.11365 & 70.72 & 14.31 & 65.59 & -135.54 \\
\hline 4 & -0.55135 & -0.17427 & 0.10800 & 0.11252 & 0.11346 & 70.61 & 14.64 & 65.65 & -135.55 \\
\hline 5 & -0.62778 & -0.18712 & 0.07949 & 0.08295 & 0.08389 & 52.05 & 9.77 & 58.09 & -96.22 \\
\hline 6 & -0.46632 & -0.16865 & 0.16527 & 0.17237 & 0.17331 & 108.16 & 24.37 & 80.95 & -214.18 \\
\hline 9 & -0.51895 & -0.11439 & 0.22159 & 0.23110 & 0.23204 & 145.02 & 34.34 & 94.92 & -292.82 \\
\hline 10 & -0.58935 & -0.15068 & 0.13631 & 0.14191 & 0.14285 & 89.05 & 19.46 & 71.91 & -174.86 \\
\hline 11 & -0.47916 & -0.10611 & 0.30732 & 0.32099 & 0.32194 & 201.43 & 49.00 & 116.69 & -410.78 \\
\hline
\end{tabular}

Table(31):Physico-chemical parameters in gas-solvation using DFT/6-31G(d,p)

\begin{tabular}{|c|c|c|c|c|c|c|c|c|c|}
\hline $\begin{array}{l}\text { DFT/6-31G(d,p) } \\
\text { Gas-Solvation }\end{array}$ & HOMO & LUMO & $\begin{array}{l}\text { Zero } \\
\text { energy }\end{array}$ & $\begin{array}{l}\text { Thermal } \\
\text { energy }\end{array}$ & $\begin{array}{l}\text { Thermal } \\
\text { enthalpy }\end{array}$ & $\begin{array}{l}\text { Total } \\
\text { energy }\end{array}$ & C.V & $S$ & $\mathrm{HF}$ \\
\hline 1 & -0.22885 & 0.00342 & 0.14477 & 0.15112 & 0.15207 & 94.83 & 25.45 & 79.32 & -326.93 \\
\hline 2 & -0.21724 & 0.09363 & 0.09224 & 0.09660 & 0.09754 & 60.62 & 13.78 & 64.68 & -135.17 \\
\hline 4 & -0.22979 & 0.09428 & 0.09232 & 0.09671 & 0.09765 & 60.69 & 14.17 & 64.86 & -135.18 \\
\hline 5 & -0.23045 & 0.09676 & 0.06367 & 0.06707 & 0.06801 & 42.09 & 9.44 & 57.38 & -95.86 \\
\hline 6 & -0.22952 & 0.09537 & 0.14933 & 0.15629 & 0.15723 & 98.07 & 23.89 & 80.20 & -213.82 \\
\hline 8 & -0.23015 & 0.07609 & 0.14812 & 0.15490 & 0.15585 & 97.20 & 25.46 & 77.29 & -213.83 \\
\hline 9 & -0.20332 & 0.08994 & 0.20567 & 0.21506 & 0.21600 & 134.95 & 33.78 & 93.61 & -292.44 \\
\hline 10 & -0.21186 & 0.08521 & 0.12046 & 0.12586 & 0.12680 & 78.98 & 18.75 & 70.72 & -174.49 \\
\hline 11 & -0.20362 & 0.08730 & 0.29092 & 0.30444 & 0.30538 & 191.04 & 48.49 & 115.39 & -410.38 \\
\hline
\end{tabular}

Table(32):Physico-chemical parameters in ion solvation using DFT/6-31G(d,p)

\begin{tabular}{|c|c|c|c|c|c|c|c|c|c|}
\hline $\begin{array}{l}\text { DFT/6-31G(d,p) } \\
\text { Ion Solvation }\end{array}$ & HOMO & LUMO & $\begin{array}{l}\text { Zero } \\
\text { energy }\end{array}$ & $\begin{array}{l}\text { Thermal } \\
\text { energy }\end{array}$ & $\begin{array}{l}\text { Thermal } \\
\text { enthalpy }\end{array}$ & $\begin{array}{l}\text { Total } \\
\text { energy }\end{array}$ & C.V & $\mathrm{S}$ & $\mathrm{HF}$ \\
\hline 1 & -0.26047 & -0.02506 & 0.15885 & 0.16626 & 0.16721 & 104.33 & 27.82 & 86.95 & -327.40 \\
\hline 2 & -0.42572 & 0.02892 & 0.10686 & 0.11133 & 0.11227 & 69.86 & 14.22 & 65.50 & -135.64 \\
\hline 3 & -0.33452 & 0.04384 & 0.27723 & 0.28886 & 0.28981 & 181.26 & 41.61 & 106.22 & -371.55 \\
\hline 4 & -0.37917 & 0.03449 & 0.10661 & 0.11109 & 0.11204 & 69.71 & 14.54 & 65.53 & -135.66 \\
\hline 5 & -0.43594 & 0.02883 & 0.07805 & 0.08151 & 0.08245 & 51.15 & 9.74 & 58.08 & -96.33 \\
\hline 6 & -0.33553 & 0.03618 & 0.16366 & 0.17074 & 0.17169 & 107.14 & 24.27 & 81.02 & -214.29 \\
\hline 7 & -0.35115 & 0.03596 & 0.13513 & 0.14091 & 0.14185 & 88.42 & 19.42 & 73.36 & -174.98 \\
\hline 8 & -0.36607 & 0.02919 & 0.16224 & 0.16916 & 0.17010 & 106.15 & 25.88 & 77.97 & -214.30 \\
\hline 9 & -0.36944 & 0.04443 & 0.22050 & 0.22993 & 0.23088 & 144.29 & 34.33 & 92.88 & -292.90 \\
\hline 10 & -0.42517 & 0.02476 & 0.13530 & 0.14085 & 0.14179 & 88.38 & 19.32 & 71.63 & -174.96 \\
\hline 11 & -0.34720 & 0.04995 & 0.30563 & 0.31843 & 0.31937 & 199.82 & 46.93 & 111.18 & -410.86 \\
\hline
\end{tabular}

Multiple linear regressions were used the $\mathrm{pKa}$ as dependent factors and the other data were independent values. 
Table (33): Predicted of pKa values from regression using DFT/STO-3G

\begin{tabular}{llllll}
\hline DFT/STO-3G & Exp. pKa & Gas phase & Ion phase & Gas Solvation & Ion Solvation \\
\hline 1 & 9.34 & 9.49 & 8.43 & 9.40 & 9.18 \\
2 & $10.60^{*}$ & 10.45 & 9.69 & 10.25 & 10.04 \\
3 & 11.25 & 11.70 & 10.03 & 11.29 & 11.07 \\
4 & 10.63 & 10.62 & 9.66 & 10.62 & 10.53 \\
5 & 10.62 & 10.77 & 10.05 & 10.69 & 10.56 \\
6 & 10.59 & 10.77 & 9.90 & 10.59 & 10.44 \\
7 & 10.53 & 10.66 & 9.73 & 10.57 & 10.49 \\
8 & 10.45 & 10.58 & 9.56 & 10.50 & 10.34 \\
9 & 10.76 & 10.78 & 9.47 & 10.75 & 10.61 \\
10 & 9.76 & 10.37 & 9.30 & 9.81 & 9.67 \\
11 & $10.65^{*}$ & 12.31 & 10.05 & 11.75 & 9.62 \\
\hline R & & 0.937 & 0.872 & 0.999 & 0.996 \\
St. error & & 0.210 & 0.294 & 0.030 & 0.051 \\
Fisher Value & & 50.30 & 22.22 & 2665.41 & 964.91 \\
$*$ Removed at regression & & & & \\
\hline
\end{tabular}

Table (34): Predicted of pKa values from regression using DFT/6-31G

\begin{tabular}{llllll}
\hline DFT/6-31G & Exp. $\mathrm{pKa}$ & Gas phase & Ion phase & Gas Solvation & Ion Solvation \\
\hline 1 & $9.34^{*}$ & 28.06 & 9.97 & 12.35 & 10.04 \\
2 & 10.60 & 10.65 & 10.66 & 10.68 & 10.65 \\
3 & 11.25 & 11.23 & 11.22 & 11.17 & 11.29 \\
4 & 10.63 & 10.51 & 10.55 & 10.57 & 10.64 \\
5 & 10.62 & 10.54 & 10.58 & 10.46 & 10.62 \\
6 & 10.59 & 10.71 & 10.58 & 10.70 & 10.59 \\
7 & 10.53 & 10.65 & 10.56 & 10.62 & 10.60 \\
8 & 10.45 & 10.49 & 10.45 & 10.44 & 10.48 \\
9 & 10.76 & 10.87 & 10.73 & 10.75 & 10.79 \\
10 & $9.76^{*}$ & 10.40 & 10.60 & 10.72 & 10.65 \\
11 & $10.65^{*}$ & 10.82 & 11.07 & 10.92 & 10.48 \\
\hline R & & 0.929 & 0.985 & 0.924 & 0.995 \\
St. error & & 0.098 & 0.046 & 0.101 & 0.026 \\
Fisher Value & 37.85 & 191.67 & 35.21 & 612.33 \\
$*$ Removed at regression & & & & \\
\hline
\end{tabular}

Table (35): Predicted of pKa values from regression using DFT/STO-3G(d,p)

\begin{tabular}{llllll}
\hline DFT/6-31G(d,p) & Exp. pKa & Gas phase & Ion phase & Gas Solvation & Ion Solvation \\
\hline 1 & 9.34 & 9.36 & 9.43 & 9.36 & 9.45 \\
2 & 10.60 & 10.61 & 10.72 & 10.66 & 10.67 \\
3 & 11.25 & 11.12 & 11.35 & 11.17 & 11.37 \\
4 & 10.63 & 10.58 & 10.59 & 10.60 & 10.66 \\
5 & 10.62 & 10.47 & 10.61 & 10.48 & 10.61 \\
6 & 10.59 & 10.75 & 10.66 & 10.75 & 10.62 \\
7 & 10.53 & 10.66 & 10.62 & 10.67 & 10.63 \\
8 & 10.45 & 10.44 & 10.53 & 10.46 & 10.52 \\
9 & 10.76 & 10.84 & 10.83 & 10.82 & 10.86 \\
10 & $9.76^{*}$ & 10.64 & 10.72 & 10.70 & 10.72 \\
11 & $10.65^{*}$ & 11.07 & 11.27 & 10.99 & 11.54 \\
\hline R & & 0.977 & 0.994 & 0.981 & 0.996 \\
St. error & & 0.113 & 0.056 & 0.103 & 0.047 \\
Fisher Value & & 148.62 & 624.68 & 180.71 & 906.32 \\
$*$ Removed at regression & & & & \\
\hline
\end{tabular}


Table (36): Predicted of pKa values from regression using HF/STO-3G

\begin{tabular}{llllll}
\hline HF/STO-3G & Exp. $\mathrm{pKa}$ & Gas phase & Ion phase & Gas Solvation & Ion Solvation \\
\hline 1 & 9.34 & 9.37 & 9.47 & 9.16 & 9.36 \\
2 & 10.60 & 10.29 & 10.49 & 10.22 & 10.26 \\
3 & 11.25 & 11.17 & 11.28 & 10.92 & 11.25 \\
4 & 10.63 & 10.56 & 10.71 & 10.48 & 10.68 \\
5 & 10.62 & 10.70 & 10.75 & 10.64 & 10.67 \\
6 & 10.59 & 10.78 & 10.70 & 10.61 & 10.55 \\
7 & 10.53 & 10.68 & 10.67 & 10.56 & 10.65 \\
8 & 10.45 & 10.33 & 10.46 & 10.18 & 10.37 \\
9 & $10.76^{*}$ & 9.87 & 10.74 & 9.85 & 10.57 \\
10 & 9.76 & 9.96 & 9.86 & 9.85 & 9.99 \\
11 & 10.65 & 10.73 & 10.91 & 10.48 & 10.68 \\
\hline R & & 0.952 & 0.983 & 0.950 & 0.960 \\
St. error & & 0.170 & 0.103 & 0.175 & 0.156 \\
Fisher Value & & 78.17 & 226.60 & 73.36 & 93.40 \\
$*$ Removed at regression & & & & \\
\hline
\end{tabular}

Table (37): Predicted of pKa values from regression using HF/6-31G

\begin{tabular}{llllll}
\hline HF/6-31G & Exp. pKa & Gas phase & Ion phase & Gas Solvation & Ion Solvation \\
\hline 1 & 9.34 & 9.24 & 9.28 & 9.46 & 9.27 \\
2 & 10.60 & 10.64 & 10.62 & 10.71 & 10.60 \\
3 & 11.25 & 11.04 & 11.16 & 11.37 & 11.16 \\
4 & 10.63 & 10.52 & 10.48 & 10.55 & 10.57 \\
5 & 10.62 & 10.42 & 10.62 & 10.61 & 10.56 \\
6 & 10.59 & 10.67 & 10.61 & 10.73 & 10.50 \\
7 & 10.53 & 10.57 & 10.52 & 10.67 & 10.54 \\
8 & 10.45 & 10.39 & 10.41 & 10.41 & 10.40 \\
9 & $10.76^{*}$ & 10.28 & 10.29 & 10.86 & 10.19 \\
10 & $9.76^{*}$ & 10.62 & 10.49 & 10.30 & 10.60 \\
11 & 10.65 & 10.53 & 10.60 & 10.81 & 10.56 \\
\hline R & & 0.977 & 0.994 & 0.984 & 0.997 \\
St. error & & 0.112 & 0.059 & 0.095 & 0.040 \\
Fisher Value & & 150.30 & 553.61 & 208.56 & 1228.39 \\
$*$ Removed at regression & & & & \\
\hline
\end{tabular}

Table (38): Predicted of pKa values from regression using HF/6-31G(d,p)

\begin{tabular}{llllll}
\hline HF/6-31G(d,p) & Exp. pKa & Gas phase & Ion phase & Gas Solvation & Ion Solvation \\
\hline 1 & $9.34^{*}$ & 13.71 & 10.26 & 7.44 & 9.88 \\
2 & 10.60 & 10.67 & 10.65 & 10.69 & 10.60 \\
3 & 11.25 & 11.15 & 11.24 & 11.16 & 11.22 \\
4 & 10.63 & 10.63 & 10.52 & 10.67 & 10.57 \\
5 & 10.62 & 10.43 & 10.64 & 10.40 & 10.58 \\
6 & 10.59 & 10.75 & 10.67 & 10.78 & 10.54 \\
7 & 10.53 & 10.64 & 10.56 & 10.65 & 10.56 \\
8 & 10.45 & 10.45 & 10.46 & 10.25 & 10.43 \\
9 & $10.76^{*}$ & 10.46 & 10.36 & 10.07 & 11.47 \\
10 & $9.76^{*}$ & 10.16 & 10.53 & 11.20 & 10.65 \\
11 & 10.65 & 10.68 & 10.68 & 10.58 & 10.62 \\
\hline R & & 0.888 & 0.973 & 0.832 & 0.993 \\
St. error & & 0.121 & 0.060 & 0.146 & 0.031 \\
Fisher Value & 22.42 & 107.91 & 13.47 & 430.61 \\
$*$ Removed at regression & & & & \\
\hline
\end{tabular}

From the table (9-38), all the regression was near to each other's and can't recognize between them to choose the best method. So, the fisher values are the best data to compare between them and choose the best method in the calculations as shown below: 


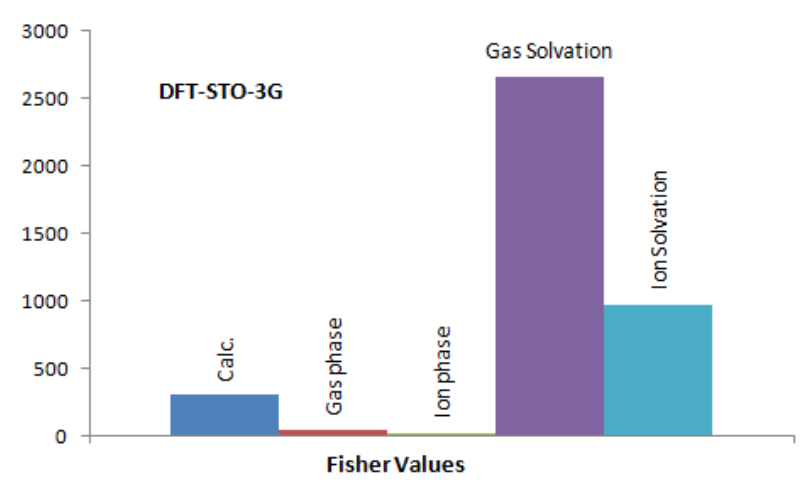

Fig. 1. Compare between the fisher data for the first method (calc.) and second method (gas, ion, gas-solvation and ion-solvation) using DFT/STO-3G

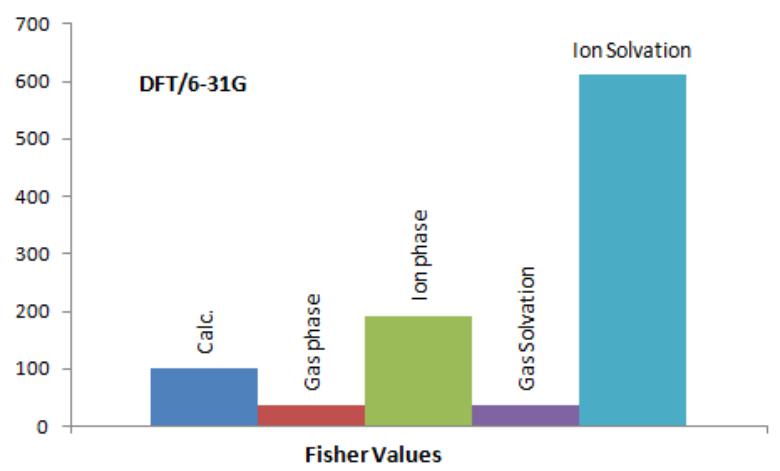

Fig. 2. Compare between the fisher data for the first method (calc.) and second method (gas, ion, gas-solvation and ion-solvation) using DFT/6-31G

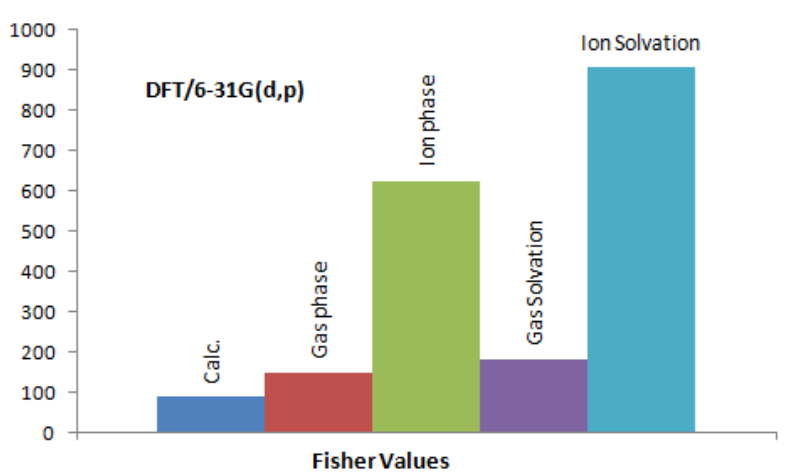

Fig. 3. Compare between the fisher data for the first method (calc.) and second method(gas, ion, gas-solvation and ion-solvation) using DFT/6-31G(d,p)

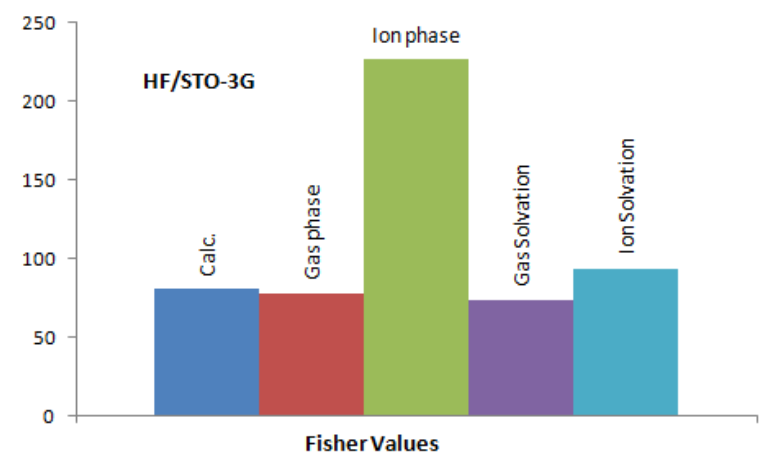

Fig. 4. Compare between the fisher data for the first method (calc.) and second method (gas, ion, gas-solvation and ion-solvation) using HF/STO-3G 


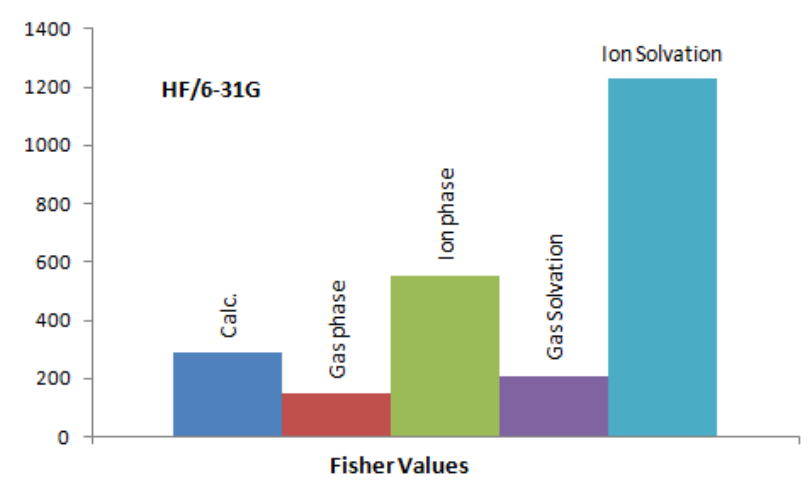

Fig. 5. Compare between the fisher data for the first method (calc.) and second method (gas, ion, gas-solvation and ion-solvation) using DFT/6-31G

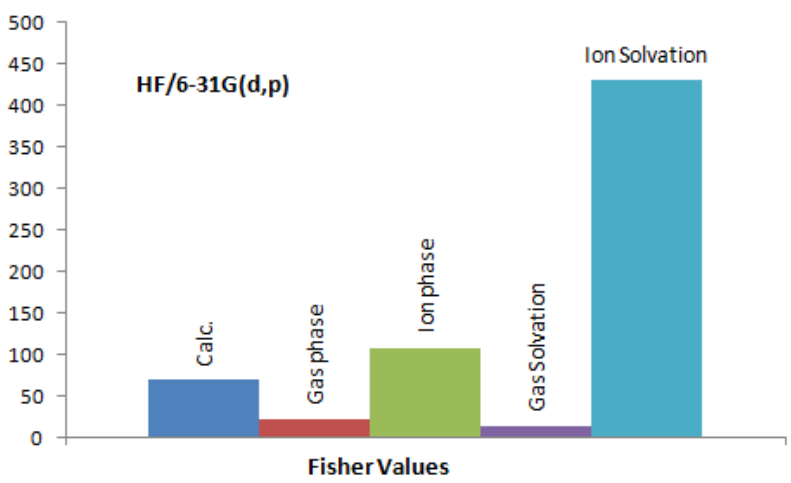

Fig. 6. Compare between the fisher data for the first method (calc.) and second method (gas, ion, gas-solvation and ion-solvation) using $\mathrm{HF} / 5-31 \mathrm{G}(\mathrm{d}, \mathrm{p})$

\section{Conclusion:}

In this research, two methods of statistics were used to evaluated and predicted $\mathrm{pKa}$ values. The first method gives an excellent predicted value of $\mathrm{pKa}$ depends on the correlation coefficient $(\mathrm{R}>0.95)$. The (DFT/STO-3G) give the best method for the determination of pKa depends on the fisher data. The second method somewhat, the ion-solvation gives excellent data for evaluated and predicted $\mathrm{pKa}$ data in four methods compare to others.

\section{References:}

[1] Liptak, M.D.; Gross, K.C.; Seybold, P.G.; Feldgus, S.; Shields, G.C.; Absolute pKa determinations for substituted phenols. J. Am. Chem. Soc., 124(22), 6421-7(2002).

[2] Liptak, M.D.; Shields, G.C.; Accurate pKa calculations for carboxylic acids using complete basis set and Gaussian-n models combined with CPCM continuum solvation methods. J. Am. Chem. Soc., 123(30), 7314-9 (2001).

[3] Zhan, C.G.; Dixon, D.A.; Absolute hydration free energy of the proton from first-principles electronic structure calculations. J. Phys. Chem. A, 105(51), 11534-40(2001).

[4] Takano, Y.; Houk, K.N.; Benchmarking the conductor-like polarizable continuum model (CPCM) for aqueous solvation free energies of neutral and ionic organic molecules. J. Chem. Theory. Comput., 1, 70-7(2005).

[5] Kelly, C.P.; Cramer, C.J.; Truhlar, D.G.; Aqueous solvation free energies of ions and ion-water clusters based on an accurate value for the absolute aqueous solvation free energy of the proton. J. Phys. Chem. B, 110(32), 16066-81 (2006).

[6] Schuurmann, G.; Cossi, M.; Barone, V.; Tomasi, J.; Prediction of the pK(a) of carboxylic acids using the ab initio continuum-solvation model PCM-UAHF. J. Phys. Chem. A, 102(33), 670612 (1998). 
[7] David C. Blakemore ; Luis C.; Ian C.; David C. Rees; Andrew W. Thomas; David M. Wilson; Anthony W.; Organic synthesis provides opportunities to transform drug discovery, Nature Chemistry, vol 10, , 383-394, (April 2018).

[8] Anjali B.; A Review on Diverse Role of Heterocyclic Moieties Containing Amino-quinoline and Azoles, Int.J.Curr.Res.Aca.Rev., 6(8): 12-39 (2018).

[9] Mossaraf H.; Ashis K. N.; A Review on Heterocyclic: Synthesis and Their Application in Medicinal Chemistry of Imidazole Moiety, Science Journal of Chemistry; 6(5): 83-94(2018).

[10] Md. Sajjad H., Farzana K. C.; Nayon U.; Laila A. B., Md. Kudrat-E-Z., Md. Masuqul H.; Synthesis, Characterization and Biological Activity Studies of Mixed Ligand Complexes with Schiff base and 2,2'-Bipyridine, International Journal of Applied Science - Research and Review, Vol.6 No.1: 2, (2018).

[11] Johnson M.;Alias A.,Janakiraman N.; Murugesan S.;Studies on Antioxidant and Phytochemical Profiles of Leptochloa uniflora Hochst, Int. Biol. Biomed. J. Autumn; Vol 1 No 4, 146-156(2015).

[12] Elena P. , Danuta Z. , Piotr G., Henryk Z., Juana F., Vitamin C, Phenolic Compounds and Antioxidant Capacity of Broccoli Florets Grown under Different Nitrogen Treatments Combined with Selenium, Pol. J. Food Nutr. Sci., Vol. 68, No. 2, pp. 179-186 (2018).

[13] Al-Ramadane O.M., Akrawi O.A., Ibrahim A.A., Al-Kazzaz A.S., National Journal of Chemistry, Volume 35, 512-520 (2009)

[14] Ammar A. Ibrahim, Asian Journal of Chemistry; vol. 23, no. 1, 215-218 (2011).

[15] Ammar A. Ibrahim, Rosiyah Y., Asian Journal of Chemistry; vol. 24, no. 6, 2634-2636 (2012).

[16] Milan R., Anna R.; Ria B., A Comparative Study of Molecular Structure, pKa, Lipophilicity, Solubility, Absorption and Polar Surface Area of Some Anti-platelet Drugs, Int. J. Mol. Sci., 17, 388; doi:10.3390/ijms17030388 (2016).

[17] Marco T.; Antonino L.; Anna M. A., Theoretical Determination of the pKa Values of Betalamic Acid Related to the Free Radical Scavenger Capacity: Comparison Between Empirical and Quantum Chemical Methods, Interdiscip Sci Comput Life Sci, DOI 10.1007/s12539-015-01013, 08 August (2015).

[18] Ammar A. Ibrahim; Eid A. Abdalrazaq, American Journal of Applied Sciences 6 (7): 13851389, (2009).

[19] Sandra B.; Alka J.M. Horvat, Dragana M. Pavlovic; Marija K. Macan, Trends in Analytical Chemistry, Vol. 26, No. 11, (2007).

[20] Christopher L. Tang; Emil A., Anna M. Pyle, Barry H. ; J. Mol. Biol.,366, 1475-1496 (2007).

[21] Raghab P.; Medhi C.; J. Chem. Sci., Vol. 116, No. 4, July, pp. 235-241 (2004).

[22] Funda T.; Murat D.; Cihan I.; Seref D.; Determination And Evaluation Of Acidity Constants Of Some Imidazole And Thiazole Linked Acetamide Compounds, Anadolu Univ. J. of Sci. and Technology-A-Appl. Sci. and Eng. 17 (2)- (2016)

[23] Peter P.; Acta Chimica Slovaca, Vol. 7, No. 1, pp. 25-30 (2014).

[24] Bishnu T.; Bernhard H. Schlegel, Density Functional Theory Calculation of pKa's of Thiols in Aqueous Solution Using Explicit Water Molecules and the Polarizable Continuum Mode, J. Phys. Chem. A, 120, 5726-5735 (2016).

[25] Mauro S.; Sandra L.; Determination of pKa Values via ab initio Molecular Dynamics and its Application to Transition Metal-BasedWater Oxidation Catalysts, Inorganics, 7, 73; doi: 10.3390/inorganics7060073 (2019).

[26] Ammar A. Ibrahim, Ghufran M. Abed, Theoretical Prediction of the Ionization Potential Using Different Methods AM1, HF and DFT International Journal of Scientific \& Engineering Research Volume 9, Issue 1, January, 726-733 (2018).

[27] Abhishek D.; Someswar C.; Physical Chemistry an Indian Journal, vol. 4, Issue 2, December (2009).

[28] Vrábel M.; Hocek M.; Havran L.; Fojta M.; Votruba I.; Klepetářová B.; Pohl R.; Eur. Jour. ofInorg. Chem., 1752 (2007).

[29] Yang X. T.; Wu H.; Ma S. J.; Hu J. J.; Wang Y.;Trans. Met. Chem., 1 (2011).

[30] Marquez-Flores Y. K.; Campos-Aldrete M. E., Salgado H. Z.; Correa-Basurto J., MelendezCamargo M. E., Med. Chem. Res., (2011).

[31] Alaattin G. ; Int. J. Mol. Sci., 6, 257-275 (2005). 